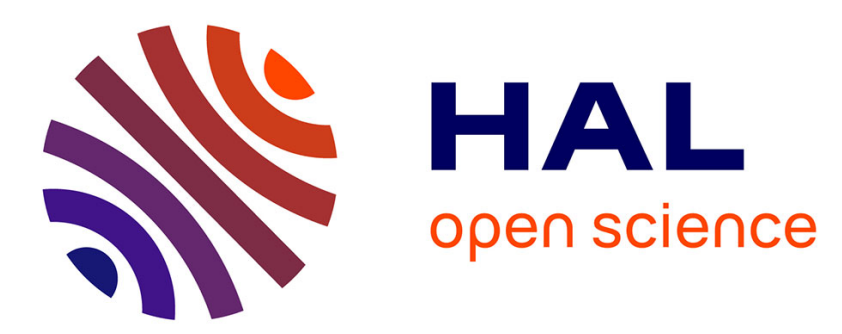

\title{
Excited-state non-adiabatic dynamics simulations of pyrrole
}

Hans Lischka, Mario Barbatti, Mario Vazdar, Mirjana Eckert-Maksic

\section{To cite this version:}

Hans Lischka, Mario Barbatti, Mario Vazdar, Mirjana Eckert-Maksic. Excited-state nonadiabatic dynamics simulations of pyrrole. Molecular Physics, 2009, 107 (08-12), pp.845-854. 10.1080/00268970802665639 . hal-00513244

\section{HAL Id: hal-00513244 \\ https://hal.science/hal-00513244}

Submitted on 1 Sep 2010

HAL is a multi-disciplinary open access archive for the deposit and dissemination of scientific research documents, whether they are published or not. The documents may come from teaching and research institutions in France or abroad, or from public or private research centers.
L'archive ouverte pluridisciplinaire HAL, est destinée au dépôt et à la diffusion de documents scientifiques de niveau recherche, publiés ou non, émanant des établissements d'enseignement et de recherche français ou étrangers, des laboratoires publics ou privés. 


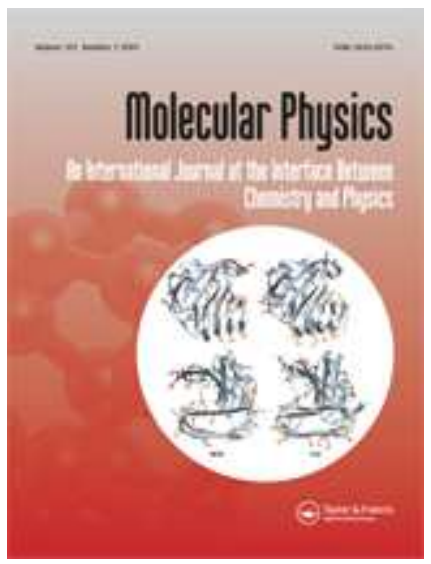

\section{Excited-state non-adiabatic dynamics simulations of pyrrole}

\begin{tabular}{|r|l|}
\hline Journal: & Molecular Physics \\
\hline Manuscript ID: & TMPH-2008-0381.R1 \\
\hline Manuscript Type: & Special Issue Paper - Fritz Schaefer \\
\hline Date Submitted by the \\
Author: & $27-$ Nov-2008 \\
\hline Keyplete List of Authors: & $\begin{array}{l}\text { Lischka, Hans; University of Vienna, Institute for theoretical } \\
\text { Chemistry } \\
\text { Barbatti, Mario; University of Vienna, Institute for Theoretical } \\
\text { Chemistry } \\
\text { Vazdar, Mario; Rudjer Bošković Institute } \\
\text { Eckert-Maksic, Mirjana; Rudjer Bošković Institute }\end{array}$ \\
\hline & \begin{tabular}{l} 
non-adiabatic dynamics, conical intersection, photochemistry \\
\hline
\end{tabular} \\
\hline
\end{tabular}

\section{S) ScholaroNE \\ Manuscript Central}




\title{
Excited-state non-adiabatic dynamics simulations of pyrrole
}

\author{
Mario Vazdar, ${ }^{\mathrm{a}}$ Mirjana Eckert-Maksić, ${ }^{\mathrm{a}^{*}}$ Mario Barbatti, ${ }^{\mathrm{b}^{*}}$ Hans Lischka ${ }^{\mathrm{b}^{*}}$ \\ ${ }^{a}$ Laboratory for Physical-Organic Chemistry - Division of Organic Chemistry and \\ Biochemistry. Rudjer Bošković Institute, 10002 Zagreb, Croatia $;{ }^{b}$ Institute for Theoretical \\ Chemistry - University of Vienna, Waehringerstrasse 17, A 1090 Vienna, Austria.
}

\begin{abstract}
Non-adiabatic on-the-fly-dynamics simulations of the photodynamics of pyrrole were performed at multireference configuration interaction level involving five electronic states with a simulation time of 200 fs. The analysis of the time dependence of the average state occupations shows that the deactivation of pyrrole to the electronic ground state takes place in about 140 fs. This deactivation time agrees very well with the experimentally measured time constant of $110 \mathrm{fs}$ for the formation of fast hydrogen atoms. After excitation into the $\mathrm{S}_{4}$ state, $80 \%$ of the trajectories followed the $\mathrm{NH}$-stretching mechanism giving rise to a population of fast $\mathrm{H}$ atoms. The computed average kinetic energy is in good accord with the experimentally observed average kinetic energy of the fast hydrogen atoms. It is found that $10 \%$ of trajectories followed the ring-puckering mechanism and 3\% followed the ring-opening mechanism. This latter mechanism was characterized in pyrrole for the first time and involves the conical intersection of lowest energy of this molecule.
\end{abstract}

Keywords: non-adiabatic dynamics; conical intersection; photochemistry; pyrrole

* Corresponding authors: H. Lischka (hans.lischka@univie.ac.at), M. Barbatti (mario.barbatti@univie.ac.at) and M. Eckert-Maksić (mmaksic@emma.irb.hr) 


\section{Introduction}

Pyrrole is one of the simplest biologically relevant heteroaromatic compounds. For this reason, its electronic states have been intensively studied both experimentally and theoretically during the last decades with the emphasis on its UV spectrum [1-10] and its photodynamics [11-24]. In particular, it is known that the deactivation of UV-excited pyrrole to the ground state occurs at a very short (femtosecond) time scale [19] with low luminescence quantum yields [25], indicating the dominance of internal conversion processes. Sobolewski and coworkers [13] have proposed that the deactivation of pyrrole and related heteroatomic compounds occurs via the $\mathrm{NH}$-stretching mechanism along a ${ }^{1} \pi \sigma_{\mathrm{NH}} *$ repulsive state. This mechanism, which has been examined in detail $[15,16,22,23]$ by means of wave packet dynamics simulations, can fully explain the presence of fast $\mathrm{H}$ atoms in the photofragmentation spectra [26]. Nevertheless, the mechanism responsible for the formation of slow $\mathrm{H}$ atoms and of other experimentally observed fragments such as $\mathrm{HCN}$ and $\mathrm{CNH}_{2}[11$, $14,17,19,20]$ is still subject of considerable debate $[15,19,26-28]$.

Recently, we have suggested [27] that non-adiabatic deactivation of pyrrole may also proceed via a ring-puckering mechanism. This second kind of mechanism could not only be the source of heavy fragments, but also partially explain the slow $\mathrm{H}$ atoms [29]. Also recently, a third deactivation mechanism that can be relevant for pyrrole was identified in thiophene [30], furan [31], imidazole [32], and in the imidazole group of adenine [33]. In this mechanism the deactivation of five-membered rings proceeds by a planar ring-opening deformation. This process was observed to occur in a minor fraction of trajectories during dynamics simulations of adenine [34]. Based on these findings, we have currently attempted and succeeded to locate this type of mechanism in pyrrole, too. 
Profant et al. [35] and Poterya et al. [28] have experimentally investigated the photolysis of pyrrole clusters. In addition, they have also performed theoretical calculations on the isolated pyrrole and on pyrrole complexes [28]. They have found that in presence of solvent molecules the $\mathrm{NH}$ stretching mechanism is inhibited, which results in a strong reduction of the fast $\mathrm{H}$ atom elimination process while keeping the slow $\mathrm{H}$ atom elimination. These are important results that on one hand once more confirm the role of the NH-stretching mechanism for the fast $\mathrm{H}$ atoms formation and on the other hand indicate that ring deformation mechanisms should be involved in the slow $\mathrm{H}$ atoms formation.

The strong dependence of different fragment yields on the excitation energy [26] indicates that the individual mechanisms are in mutual competition and can play different roles depending on the initial conditions. Excited-state energy surfaces have been investigated in detail under special consideration of crossings between different energy surfaces as already mentioned above $[1,18,23,27,28,36]$ and reaction paths have been constructed subsequently. This information led to substantial progress in the understanding of the photochemical processes in pyrrole. However, it turned out to be very difficult to estimate the importance of individual intersections and related reaction pathways. In order to better understand how these mechanisms are activated, it is desirable to perform dynamics simulations. Such simulations exhibit a substantial complexity. For instance, as for selecting the proper quantum chemical methods, it needs to be taken into account that: first, the nonadiabatic dynamics of pyrrole involves multiple excited states showing often multireference character and, secondly, that it is essentially impossible to identify just a few important internal degrees of freedom by which the photochemical reaction mechanism can be described. Therefore, an essential condition is the usage of the full set of nuclear coordinates. These are usual requirements to be met e.g. in simulations of organic chromophores exhibiting high density of excited states $[34,37,38]$. One convenient way to satisfy especially 
the second condition is to use mixed-quantum classical dynamics methods [39-44]. In this work surface hopping dynamics is performed using the fewest-switches algorithm of Tully [45]. The advantage of this approach is that it allows the application of an "on-the-fly" strategy $[42,43,46]$ where a pre-selection of certain internal degrees of freedom and any fitting of pre-computed potential energy points is avoided by computing at each time step the energies, the complete energy gradient and non-adiabatic coupling terms required for the integration of Newton's equations of motion and the time-dependent Schrödinger equation. This on-the-fly strategy is computationally very expensive and requires analytical energy gradients and non-adiabatic coupling vectors for computational efficiency. Due to the stringent computational requirements most of the photodynamical simulations have been performed so far at the relatively cost-effective complete active space self consistent field level (CASSCF). Since in this case dynamical electron correlation effects are mostly neglected, the relative balance of electronic states of different character can be strongly violated. It should be stressed that the non-adiabatic dynamics simulations presented here were carried out at a significantly higher level using the MR-CISD method including five electronic states. This represents the state-of-the-art approach for this kind of simulations, which has not been documented before for molecules of the size of pyrrole to the best of our knowledge. The present calculations have been made possible by use of the analytic gradient features of the program package COLUMBUS [47-49] as it will be described below.

\section{Computational details}

Multireference configuration interaction (MRCI) and complete active space self-consistent field (CASSCF) calculations were performed for pyrrole. The CAS space was comprised of four $\pi$ electrons in five orbitals (two $\pi$ orbitals, two $\pi^{*}$ orbitals and one Rydberg $3 \mathrm{~s}$ orbital). This space will be conventionally designated as $\operatorname{CAS}(4,5)$ in the text. State averaging was 
performed over five singlet states with equal weights (ground state, two valence $\pi \pi^{*}$ states and two Rydberg $\pi 3 \mathrm{~s}$ states), which will be denoted as SA-5. MRCI calculations were performed based on the orbitals computed by the $\operatorname{SA}-5-\operatorname{CASSCF}(4,5)$ wave function. The reference configurations for the MRCI were constructed within the CAS $(4,5)$ by allowing single and double excitations from the two $\pi$ orbitals into the two $\pi^{*}$ orbitals and the Rydberg 3s orbital. The final configuration space was constructed by allowing all single and double excitations from the reference configurations into the virtual orbital space (MR-CISD). All core electrons and the lowest eight additional orbitals were frozen in the MRCI calculations and the interacting space restriction [50] was applied. The basis set was composed of aug'-ccpVDZ type [51] on the nitrogen and carbon atoms (the prime indicates that d-aug functions were removed). On the hydrogen atom connected to nitrogen, the cc-pVDZ basis set was used, whereas for the remaining hydrogen atoms the cc'-pVDZ basis set was used (the prime signifies that $\mathrm{p}$-functions were deleted). This hybrid basis set will be denoted as BS.

The MRCI approach and the basis set were selected by balancing the accuracy requirements of the calculations of four excited states of different character (see Table 1) and the need for computational efficiency, since an on-the-fly approach requires several tens of thousands of individual MRCI calculations to be carried out. Therefore, before starting the dynamics simulations an extensive set of calculations had been performed, including the Franck-Condon region, the seam of conical intersections, and reaction pathways. For the determination of minima on the crossing seam (MXS), starting geometries were selected from our previous MRCI calculations on pyrrole [27] and were reoptimized with the abovedescribed MRCI method. Reaction paths for the two ring-deformation processes were constructed by the method of linear interpolation of internal coordinates (LIIC) between the ground-state geometry and the corresponding ring-deformed conical intersections. The reaction path for the $\mathrm{NH}$-stretching process was constructed by rigidly stretching the $\mathrm{NH}$ 
distance in steps of $0.2 \AA$ starting from the ground state equilibrium structure up to a $\mathrm{NH}$ distance of $2.6 \AA$.

All energy calculations and MXS optimizations were performed by using analytical gradient and non-adiabatic coupling procedures described in References [52-56]. For vertical excitation energy calculations, the Davidson correction $(+Q)[53,57,58]$ was used in order to describe higher order excitation effects. For the $\mathrm{C}_{2 \mathrm{v}}$ labeling of the states, the $x$ axis was assumed to be oriented perpendicular to the ring plane.

Mixed quantum-classical dynamics calculations were performed for pyrrole by using an on-the-fly approach $[42,43,46,59,60]$. Energies, gradients, and non-adiabatic coupling vectors were computed at each time step at the MR-CISD/SA-5-CASSCF(4,5)/BS level of theory. The nuclear motion was represented by classical trajectories computed by numerical integration of Newton's equations by the velocity-Verlet algorithm [61]. Non-adiabatic effects were taken into account by means of the surface hopping approach [45]. Time-dependent adiabatic populations were corrected for decoherence effects [62] ( $\alpha=0.1$ hartree) and used to calculate surface hopping probabilities in accordance to the Tully's fewest switches approach [45]. In order to alleviate the computational costs, no coupling vectors were calculated between non-consecutive states [44]. In total, 90 trajectories were computed. The initial Cartesian coordinates and momenta were selected from a quantum harmonic oscillator (Wigner) distribution in the ground state. The trajectories were started in the $\mathrm{S}_{4}$ state at these geometries. This procedure gave rise to a composition of $60 \%$ of trajectories initially in the $\pi \pi^{*}$ states and $40 \%$ in the $\pi 3 \mathrm{~s} / \pi \sigma_{\mathrm{NH}} *$ states. The minimum excitation energy was $6.36 \mathrm{eV}$ while the average was $6.76 \mathrm{eV}$ with a standard deviation of $0.26 \mathrm{eV}$. The trajectories were then propagated for a maximum time of $200 \mathrm{fs}$ with a time step of $0.5 \mathrm{fs}$.

The structures of the puckered geometries were described in terms of the CremerPople parameters $\mathrm{Q}$ and $\phi[63]$. While the parameter $\mathrm{Q}$ measures the extent of puckering $(\mathrm{Q}=$ 
Table 1. (around here)

\section{Analysis of the energy surfaces}

In order to investigate the reliability of the MRCI method used in the dynamics study, we have performed a series of tests and comparisons with other previously published results. Specifically, we have compared vertical excitation energies, reaction paths, and MXS structures with results obtained with methods of higher level of theory.

\subsection{Vertical excitation energies}

The theoretical computation of vertical excitation energies of pyrrole and the assignment of the experimental UV spectrum have been a matter of discussion for a long period of time [1, $3,4,8,9]$. The currently calculated values are compared to other available theoretical and experimental results in Table 1. The comparison reveals that vertical excitation energies computed by the MR-CISD/SA-5-CASSCF(4,5)/BS method are in good accordance with 
results obtained previously by the MR-CISD+Q/SA-5-CAS(6,5)+AUX(1)/d-aug-cc-pVDZ method [27] where the auxiliary (AUX) orbital represents the 3s Rydberg orbital into which single excitations from the valence $\operatorname{CAS}(6,5)$ are allowed. Most of the calculated vertical excitation energies differ by ca. $0.1-0.2 \mathrm{eV}$, except in the case of the ${ }^{1} \mathrm{~B}_{1}$ state where this difference is $0.35 \mathrm{eV}$. Furthermore, the present results for the ${ }^{1} \mathrm{~A}_{2}$ and ${ }^{1} \mathrm{~B}_{1}$ Rydberg states are in excellent agreement with experimental values assigned in Ref. [9]. The current energies of the $\pi \pi^{*}$ valence states are higher than in most of the other methods with the deviation being particularly large in comparison to the CASPT2 results. Nevertheless, a series of different methods, like MRCI [2, 4], EOM-CCSD [8], CC3 [3], and TDDFT [4] indicates that CASPT2 might be underestimating these transition energies. Therefore, we conclude that the current MRCI approach is adequate for calculation of vertical excitation energies.

Fig. 1 (around here)

\subsection{Conical intersections}

In Fig. 1 the MXS structures between ground state and the $S_{1}$ state are presented. The comparison of selected geometrical parameters for the ring-puckered (Fig. 1a) and the NHstretched (Fig. 1b) MXS structures reveals that they are in very good agreement with the benchmark MRCI values [27].

In Fig. 1a, the MXS between the valence $\pi \pi^{*}$ state and the ground state shows an out-ofplane deformation with strong stretching of one of the $\mathrm{CN}$ bonds. We shall refer to this conical intersection as the ring-puckered MXS. The values of dihedral $\mathrm{CCCN}$ and $\mathrm{CCCH}$ dihedral angles are very close to the benchmark ones, being only by ca. $2^{\circ}$ smaller. The length of the broken $\mathrm{CN}$ bond is $1.607 \AA$, thus being $0.007 \AA$ shorter than the value obtained with the benchmark method. In Fig. 1b, the NH-stretched MXS is shown. It arises from the crossing 
between the ground state and the lowest $\pi \sigma_{\mathrm{NH}}{ }^{*}$ state. In comparison to the benchmark MRCI value, the NH distance using the current method is shorter by $0.007 \AA$.

As mentioned in the Introduction, based on previous findings for other five-membered heteroaromatic molecules [30, 31, 33], we have searched for a planar ring-opened MXS in pyrrole as well. The optimized structure, obtained at the MRCI level of theory, is presented in Fig. 1c. It should be pointed out that the MXS is planar and that the CN distance is $2.512 \AA$, which is by about $0.9 \AA$ longer than the $\mathrm{CN}$ distance observed in the ring puckered MXS (Fig. 1a). It is important to note that the ring-opened MXS is the lowest energy conical intersection identified in pyrrole so far and it arises from the crossing between the $\pi \sigma_{\mathrm{NC}}{ }^{*}$ state and the ground state.

Table 2. (around here)

Although the similarity of geometrical parameters suggests that the selected MRCISD/SA-5-CASSCF(4,5)/BS level of theory is adequate, it is also of importance to compare the energies of the MXSs. MRCI and MRCI+Q energy values of pyrrole MXSs obtained by the MR-CISD(Q)/SA-5-CASSCF(4,5)/BS and benchmark MRCI values [27] are summarized in Table 2. The analysis of presented data shows that the energies of the MXSs are in very good agreement with the benchmark ones. The comparison among results reveals that the selected MRCI method is well suited for the description of both ring-puckering and NHstretching mechanisms. In particular, the current MRCI and MRCI+Q energies of the ringpuckered MXS are by $0.06 \mathrm{eV}$ higher and $0.07 \mathrm{eV}$ lower than the benchmark MRCI and MRCI+Q values, respectively. For the $\mathrm{NH}$-stretched MXS, the MRCI energy is by $0.04 \mathrm{eV}$ higher, whereas the MRCI+Q value is by $0.18 \mathrm{eV}$ lower than the benchmark values. 


\subsection{Reaction paths}

We have computed the reaction pathways between the ground state minimum and the three MXSs described in the previous section using the MR-CISD/SA-5-CASSCF(4,5)/BS level of theory. The resulting potential energy curves are shown in Fig. 2.

Fig. 2 (around here)

Comparison with the results obtained with the MR-CISD+Q/SA-5-
CAS(6,5)+AUX(1)/d-aug-cc-pVDZ method (Fig. 2 in [27]) reveals that NH-stretching potential energy curves (Fig. 2a) agree very well for the ground state $S_{0}$ and the two Rydberg ${ }^{1} \mathrm{~A}_{2}$ and ${ }^{1} \mathrm{~B}_{1}$ states for the whole range of $\mathrm{NH}$ distances. The main difference is that the crossing between the lowest Rydberg state and the ground state occurs at around $1.9 \AA$ in the present work instead of $2.1 \AA$ found in our earlier study [27]. The other features of the potential energy curves exhibit the same behavior as observed earlier. Specifically, the lowest two Rydberg states show small energy barriers $\left(0.24 \mathrm{eV}\right.$ for the ${ }^{1} \mathrm{~A}_{2}$ state and 0.12 for the ${ }^{1} \mathrm{~B}_{1}$ state) at the NH distance of $1.2 \AA$ necessary to transform the $\pi 3 \mathrm{~s}$ orbital into the $\pi \sigma_{\mathrm{NH}}{ }^{*}$ state as expected for a stretching of the $\mathrm{NH}$ bond. It should also be pointed out that the two valence ${ }^{1} \mathrm{~A}_{1}$ and ${ }^{1} \mathrm{~B}_{2}$ states show the same energy profile until the NH distance of $1.8 \AA$. After that, an intrusion of higher excited states occurs (not shown), which is presumably a direct consequence of the CAS $(4,5)$ active space. However, in the NH stretching mechanism, the deactivation occurs via conical intersections among Rydberg states and the ground state and the differences in the valence states for large NH distances are of minor importance.

Fig. $2 b$ shows that the LIIC path of the ring-puckering mechanism in the $\pi \pi^{*}$ state occurs without barrier. Indeed, it is clearly seen that the lowest $\pi \pi^{*}$ state is diabatically connected to the ground state, which may make it especially efficient for the internal conversion. The same result was observed in our previous study [27] thus providing additional 
support for using the applied method. In the case of the ring-opening mechanism Fig. 2c shows that the initially excited $\pi \pi^{*}$ states can deactivate without barrier along this pathway. The character of the state should, however, change into $\pi \sigma_{\mathrm{NC}} *$ in order to lead to the crossing with the ground state.

Apart from the fact that the $\mathrm{NH}$-stretching mechanism should dominate at low excitation energies, it is difficult to draw general conclusions about the efficiency of each mechanism based on the reaction paths alone in a clear cut way. When the excitation leads into the spectral region of the $\pi \pi^{*}$ state all mechanisms are energetically possible. In favor of the $\mathrm{NH}$-stretching mechanism is the fact that it requires the smallest deformations from the Franck-Condon region in terms of mass-weighted distances (see Fig. 2). On the other hand, it also requires the diabatic transformation from the $\pi \pi^{*}$ state into the $\pi \sigma_{\mathrm{NH}}{ }^{*}$ state, which depends upon the activation of out-of-plane modes $[18,27]$. The ring-opening mechanism involves the lowest energy conical intersection, but it requires the largest deformations from the Franck-Condon region and diabatic changes in the wave function at the same time. Finally, the ring-puckering mechanism, as already mentioned, can directly proceed through a diabatic connection. However, it involves the highest energy portions of the seam of conical intersections.

\section{Dynamics simulations of pyrrole}

The non-adiabatic excited state dynamics of pyrrole was started from the $\mathrm{S}_{4}$ state thus making all pathways discussed in the previous section energetically available. The resulting average adiabatic populations of the ground and excited states as a function of time are presented in Fig. 3. Their analysis shows that the $\mathrm{S}_{4}$ state transfers its population to the $\mathrm{S}_{3}$ state in the first $10 \mathrm{fs}$. After ca. $50 \mathrm{fs}$, the $\mathrm{S}_{4}$ state is almost completely depopulated. The populations of $\mathrm{S}_{3}$ and $\mathrm{S}_{2}$ states reach a maximum at $10 \mathrm{fs}$ and $20 \mathrm{fs}$, respectively. At about $75 \mathrm{fs}$, these states are 
already depopulated. The $\mathrm{S}_{2}$ state shows a repopulation between 100 and $150 \mathrm{fs}$. The population of the $S_{1}$ state increases reaching a maximum at 75 fs. At 100 fs, the $S_{1}$ and $S_{0}$ states have approximately the same population. Between $100 \mathrm{fs}$ and $200 \mathrm{fs}$, the simulation is basically reduced to the $S_{1} / S_{0}$ two-state dynamics, with the complete population transferred to the ground state at about 200 fs.

Fig. 3 (around here)

The $\mathrm{S}_{1}$ population shows a consecutive two-step first order decay type of behavior. By fitting the $\mathrm{S}_{1}$ population curve with the function

$$
f(t)=\frac{\tau_{2}}{\tau_{2}-\tau_{1}}\left[\exp \left(-\frac{t}{\tau_{1}}\right)-\exp \left(-\frac{t}{\tau_{2}}\right)\right]
$$

two time constants $\tau_{1}=44 \pm 2$ fs and $\tau_{2}=80 \pm 2$ fs are obtained. Here, $\tau_{1}$ measures the population of $S_{1}$ from the collection of states $S_{4}$ to $S_{2}$ and $\tau_{2}$ describes the depopulation $\mathrm{S}_{1} \rightarrow \mathrm{S}_{0}$. The approximate time constant for the overall population of the ground state can be obtained by fitting the $\mathrm{S}_{0}$ population with the function

$$
f(t)=1-\exp \left(-\frac{t}{\tau_{0}}\right)
$$

which gives $\tau_{0}=139 \pm 2$ fs. Note that in these three time constants the error bars denote the uncertainty of the fitting procedure and not of the process itself, which certainly is larger than a few femtoseconds.

In Fig. 4 a summary of the results of the dynamics simulation in terms of the fraction of trajectories following each of the three mechanisms is given. The NH-stretching is the main mechanism after excitation of pyrrole to the $\mathrm{S}_{4}$ state. This mechanism occurs in $80 \%$ of the trajectories. Other $13 \%$ follow ring-deformation mechanisms (ring-opening and ring- 
puckering). $7 \%$ do not deactivate within the $200 \mathrm{fs}$ of the dynamics simulation. Because of the uncertainties associated to the dynamics simulations and to the relatively small number of trajectories, these fractions should be taken as qualitative trends of occurrence of each mechanism, rather than a quantitative assessment of them. If trajectories starting in the $\pi \pi^{*}$ and in the $\pi \sigma_{\mathrm{NH}}{ }^{*}$ states are independently analyzed, these fractions remain essentially the same, implying that the population of each mechanism depends on the excitation energy, but not on the nature of the state. The fact that the fast $\mathrm{H}$ atom is formed along the $\mathrm{NH}$ stretching pathway either excited in the $\pi \pi^{*}$ or $\pi \sigma^{*}$ states has also been observed in the photofragment translational spectroscopy studies by Cronin et al. [26].

Experimental pump of pyrrole with $250 \mathrm{~nm}(4.96 \mathrm{eV})$ laser pulse followed by ionization probe with $241 \mathrm{~nm}(5.15 \mathrm{eV})$ pulse reveals two time constants, $\tau_{\mathrm{f}}=110 \pm 80 \mathrm{fs}$ and $\tau_{\mathrm{s}}=1100 \pm 500$ fs [19]. These time constants correspond to the time for formation of fast and slow $\mathrm{H}$ atoms, respectively. Since most of trajectories in our simulations finished in the ground state of the dissociated pyrrolyl $+\mathrm{H}$ system, the deactivation time $\tau_{0}$ should also approximately give the time for the formation of the fast $\mathrm{H}$ atoms population. Indeed, the comparison of $\tau_{0}$ and $\tau_{\mathrm{f}}$ shows good agreement. Note, however, that the initial state in the experiments (low energy $\pi \sigma_{\mathrm{NH}^{*}}$ ) and in the simulations (high energy $\pi \pi^{*}$ and $\pi \sigma_{\mathrm{NH}}{ }^{*}$ ) are not the same. This is an indication that the fast $\mathrm{H}$ elimination occurs directly by the same process, as soon as there is enough energy to overcome the $\pi 3 \mathrm{~s} / \pi \sigma_{\mathrm{NH}} *$ barrier in the $\mathrm{S}_{1}$ state.

Fig. 4 (around here)

Fig. 5 (around here)

The analysis of $\mathrm{NH}$ and $\mathrm{CN}$ bond distances was conducted for all trajectories and the results are presented in Fig. 5. The top panel of this figure shows that in some cases the CN distance is elongating during the dynamics. This behavior can be ascribed to the ring-opening 
and ring-puckering deactivation mechanisms. Since the main deactivation channel is the NHstretching, the majority of trajectories do not exhibit elongation of this specific bond. In the bottom panel of Fig. 5 the NH distance is monitored. In this figure three kinds of trajectories can be distinguished. For part of the trajectories the $\mathrm{NH}$ distance remain constant at about 1 $\AA$. They correspond to the trajectories following ring-distortion mechanisms. A minor fraction of trajectories (3) has the $\mathrm{NH}$ distance oscillating at a medium distance of about 2 or $3 \AA$. These are cases where the NH-stretching mechanism is activated, but instead finishing in dissociation, the hot ground state of pyrrole is formed. In most of the trajectories the $\mathrm{NH}$ distance is steadily increasing. In these cases, the NH-stretching mechanism is activated and the $\mathrm{H}$ atom elimination is taking place. It should be mentioned that a cut-off value of $10 \AA$ for NH distance was used in Fig. 5 in order to simplify the data analysis. In some of the trajectories, however, the NH distance was longer, up to $40 \AA$.

Fig. 6 (around here)

Fig. 6a shows that the hydrogen dissociation starts on average 54 fs after the photoexcitation. The kinetic energy of the dissociated hydrogen atom has a broad distribution around the average value of $1.2 \mathrm{eV}$ (Fig. 6b). This value is in very good agreement with the experimental results, $\sim 1 \mathrm{eV}[11,26]$, for the center of the fast $\mathrm{H}$-elimination peak in the kinetic energy release spectra. Note that, as expected, there is no formation of a slow Helimination peak, which should take place in the picosecond timescale [19], much longer than the maximum simulation time (200 fs). The NH distance at the $\mathrm{S}_{1} \rightarrow \mathrm{S}_{0}$ hopping time is shown in Fig. 6c for all trajectories that have returned to the ground state. The histogram shows two distinct peaks. The first peak with average at $1.0 \AA$ will be discussed below. The second peak starts at $1.5 \AA$ and presents a long tail for large distances up to $4 \AA$. This peak corresponds to the trajectories deactivated by means of the NH-stretching mechanism. Its average value at 
$2.1 \AA$ is $0.2 \AA$ larger than the NH distance for the crossing between the lowest $\pi \sigma^{*}$ state and the ground state shown in Fig. 2 (left panel).

Fig. 7 (around here)

Twelve out of ninety trajectories did not follow the NH-stretching mechanism. They appear in the short-distance peak in Fig. 6c. In order to understand which kind of mechanism they followed, it is useful to project them on the Cremer-Pople (CP) space Q- $\phi$. This is shown in Fig. 7 for all structures for which the $\mathrm{S}_{1}-\mathrm{S}_{0}$ energy gap is smaller than $0.5 \mathrm{eV}$ (open dots) and for structures at the hopping time (full dots). The ring-opened MXS is at $\mathrm{Q}=0 \AA$ and the ring-puckered MXS is shown by a cross ( $E_{1}$ conformation). Since the ring-opened and the ring-puckered conical intersections correspond to distinct types of structures on the crossing seam with different electronic configurations, it could be expected that the structures at the hopping time would cluster in two disjoint regions around these MXSs. This, however, is not the case. Fig. 7 shows that the non-adiabatic events occur in a large continuous portion of the CP space, indicating that the crossing seam spans this entire region. The degree of puckering varies from almost planar $(\mathrm{Q}=0.15 \AA)$ to the strongly puckered structures $(\mathrm{Q}=0.75 \AA)$. Most of hopping events occur at $\mathrm{E}_{1},{ }^{2} \mathrm{~T}_{1}$ and ${ }^{2} \mathrm{E}$ conformations, indicating that not only the $\mathrm{E}_{1}$ conformation of the ring-puckered MXS, but also other kinds of puckering can give rise to conical intersections in pyrrole.

If we take $\mathrm{Q}=0.3 \AA$ as an arbitrary threshold to distinguish between the ring-opening and ring-puckering mechanisms, nine trajectories deactivated at ring-puckered conformations and three trajectories deactivated at ring-opened conformations, thus corresponding to $10 \%$ and $3 \%$ of the total number of trajectories, respectively (see Fig. 4). 


\section{Conclusions}

The photochemical processes in pyrrole were investigated using a high-level multireference configuration interaction method (MRCI) giving a balanced description of the four studied excited states, two of Rydberg character and two valence states. Cuts along the potential energy surfaces connecting the Franck-Condon region and three different minima on the crossing seam (MXS) (NH dissociation, ring puckering, and a planar ring-opened MXS) describe possible deactivation pathways. One of these intersection points, the ring-opened MXS, was characterized for the first time. Although it is the conical intersection of the lowest energy identified in pyrrole so far, its efficiency for the internal conversion process seems to be reduced by the required strong geometric deformations and by the diabatic change of the initially excited $\pi \pi^{*}$ state into the $\pi \sigma_{\mathrm{NC}} *$ state, which in turn crosses the ground state.

Non-adiabatic surface-hopping dynamics simulations of pyrrole were performed for 200 fs starting in the $\mathrm{S}_{4}$ state and using a high-level MR-CI approach for the electronic structure calculations. The dynamics simulations show that in fact all three types of conical intersections were accessed. The transfer of population from the initially excited $\mathrm{S}_{4}$ state to the ground state takes place in about 140 fs. This process occurs basically in two steps, with the $\mathrm{S}_{1}$ state being populated in about $44 \mathrm{fs}$ and then being depleted in about $80 \mathrm{fs}$. Most of trajectories $(80 \%)$ dissociated rapidly along the repulsive $\pi \sigma_{\mathrm{NH}^{*}} *$ state giving rise to a population of fast $\mathrm{H}$ atoms. The computed deactivation time of 140 fs agrees very well with the experimentally measured time constant of $110 \mathrm{fs}$ for the formation of fast hydrogen atoms. The computed average kinetic energy agrees very well with the experimentally observed average kinetic energy of the fast hydrogen atoms. A fraction of $13 \%$ of trajectories follows ring-deformation channels involving either ring puckering $(10 \%)$ or planar ring opening $(3 \%)$. These fractions did not depend on whether the initial state had $\pi \pi^{*}$ or $\pi \sigma_{\mathrm{NH}} *$ character. 
Our calculations provide a detailed picture of the photodeactivation processes in pyrrole. Although the main objective of this work - the observation of the occurrence of the different deactivation mechanisms - has been accomplished, it should be noted that the participation of $\pi \sigma_{\mathrm{NH}}{ }^{*}$ states in the initial conditions was much higher than what would be expected from the oscillator strengths of these two transitions. This bias occurred because of the relatively high vertical excitation energy of the ${ }^{1} B_{1}$ Rydberg state, which caused frequent exchange of position with the $\pi \pi^{*}$ in the Wigner sample. Interestingly, it turned out that the observed percentages of the different mechanisms was insensitive to the initial character of $\mathrm{S}_{4}$, consequently implying that that this bias is not so critical for the general interpretations. Nevertheless, more investigations are needed to analyze the influence of excitation energies on the product yields in order to explain the experimentally observed strong energy dependence of the branching ratios for fast and slow hydrogen atoms.

\section{Acknowledgments}

This work was supported by the Austrian Science Fund within the framework of the Special Research Program F16 (Advanced Light Sources) and Project P18411-N19. The calculations were partially performed at the Linux PC cluster Schrödinger III of the computer center of the University of Vienna. The work in Zagreb (M.E.M and M.V.) was supported by the Ministry of Science, Education and Sport through the project 098-0982933-2920 and the COST D37 action.

\section{References}

[1] L. Serrano-Andrés, M. Merchán, I. Nebotgil, B. O. Roos, and M. Fulscher, J. Am. Chem. Soc. 115, 6184 (1993).

[2] M. H. Palmer, I. C. Walker, and M. F. Guest, Chem. Phys. 238, 179 (1998). 
[3] O. Christiansen, J. Gauss, J. F. Stanton, and P. Jorgensen, J. Chem. Phys. 111, 525 (1999).

[4] D. J. Tozer, R. D. Amos, N. C. Handy, B. O. Roos, and L. Serrano-Andres, Mol. Phys. 97, 859 (1999).

[5] J. Wan, J. Meller, M. Hada, M. Ehara, and H. Nakatsuji, J. Chem. Phys. 113, 7853 (2000).

[6] B. O. Roos, P. A. Malmqvist, V. Molina, L. Serrano-Andres, and M. Merchan, J. Chem. Phys. 116, 7526 (2002).

[7] C. G. Zhan, and D. A. Dixon, J. Mol. Spectrosc. 216, 81 (2002).

[8] P. Celani, and H. J. Werner, J. Chem. Phys. 119, 5044 (2003).

[9] M. H. Palmer, and P. J. Wilson, Mol. Phys. 101, 2391 (2003).

[10] M. Pastore, C. Angeli, and R. Cimiraglia, Chem. Phys. Lett. 422, 522 (2006).

[11] D. A. Blank, S. W. North, and Y. T. Lee, Chem. Phys. 187, 35 (1994).

[12] A. B. Trofimov, H. Köppel, and J. Schirmer, J. Chem. Phys. 109, 1025 (1998).

[13] A. L. Sobolewski, W. Domcke, C. Dedonder-Lardeux, and C. Jouvet, PCCP 4, 1093 (2002).

[14] J. Wei, A. Kuczmann, J. Riedel, F. Renth, and F. Temps, PCCP 5, 315 (2003).

[15] V. Vallet, Z. G. Lan, S. Mahapatra, A. L. Sobolewski, and W. Domcke, Faraday Discuss. 127, 283 (2004).

[16] V. Vallet, Z. G. Lan, S. Mahapatra, A. L. Sobolewski, and W. Domcke, J. Chem. Phys. 123 (2005).

[17] J. Wei, J. Riedel, A. Kuczmann, F. Renth, and F. Temps, Faraday Discuss. 127, 267 (2004).

[18] H. Köppel, E. V. Gromov, and A. B. Trofimov, Chem. Phys. 304, 35 (2004).

[19] H. Lippert, H. H. Ritze, I. V. Hertel, and W. Radloff, Chemphyschem 5, 1423 (2004).

[20] A. J. van den Brom, M. Kapelios, T. N. Kitsopoulos, N. H. Nahler, B. Cronin, and M. N. R. Ashfold, PCCP 7, 892 (2005).

[21] I. Frank, and K. Damianos, Journal of Chemical Physics 126 (2007). 
[22] Z. Lan, A. Dupays, V. Vallet, S. Mahapatra, and W. Domcke, Journal of Photochemistry and Photobiology a-Chemistry 190, 177 (2007).

[23] Z. Lan, and W. Domcke, Chem. Phys. 350, 125 (2008).

[24] A. Kumar, M. Kolaski, and K. S. Kim, J. Chem. Phys. 128 (2008).

[25] E. J. Shin, Bull. Korean Chem. Soc. 25, 907 (2004).

[26] B. Cronin, M. G. D. Nix, R. H. Qadiri, and M. N. R. Ashfold, PCCP 6, 5031 (2004).

[27] M. Barbatti, M. Vazdar, A. J. A. Aquino, M. Eckert-Maksic, and H. Lischka, J. Chem. Phys. 125, 164323 (2006).

[28] V. Poterya, V. Profant, M. Farnik, P. Slavicek, and U. Buck, J. Chem. Phys. 127, 064307 (2007).

[29] M. Barbatti, B. Sellner, A. J. A. Aquino, and H. Lischka, in Radiation Induced Molecular Phenomena in Nucleic Acid, edited by M. K. Shukla, and J. Leszczynski (Springer, Netherlands, 2008), pp. 209.

[30] S. Salzmann, M. Kleinschmidt, J. Tatchen, R. Weinkauf, and C. M. Marian, PCCP 10, 380 (2008).

[31] N. Gavrilov, S. Salzmann, and C. M. Marian, Chem. Phys. 349, 269 (2008).

[32] M. Barbatti, H. Lischka, S. Salzmann, and C. M. Marian, J. Chem. Phys., submitted (2008).

[33] S. Perun, A. L. Sobolewski, and W. Domcke, Chem. Phys. 313, 107 (2005).

[34] M. Barbatti, and H. Lischka, J. Am. Chem. Soc. 130, 6831 (2008).

[35] V. Profant, V. Poterya, M. Farnik, P. Slavicek, and U. Buck, J. Phys. Chem. A 111, 12477 (2007).

[36] A. L. Sobolewski, and W. Domcke, Chem. Phys. 259, 181 (2000).

[37] I. Antol, M. Vazdar, M. Barbatti, and M. Eckert-Maksic, Chem. Phys. 349, 308 (2008).

[38] M. Barbatti, M. Ruckenbauer, J. J. Szymczak, A. J. A. Aquino, and H. Lischka, PCCP 10, 482 (2008). 
[39] J. C. Tully, Faraday Discuss. 110, 407 (1998).

[40] A. Ferretti, G. Granucci, A. Lami, M. Persico, and G. Villani, J. Chem. Phys. 104, 5517 (1996).

[41] N. L. Doltsinis, and D. Marx, Phys. Rev. Lett. 88, 166402 (2002).

[42] M. Barbatti, G. Granucci, M. Persico, M. Ruckenbauer, M. Vazdar, M. Eckert-Maksic, and H. Lischka, J. Photochem. Photobiol., A 190, 228 (2007).

[43] E. Fabiano, T. W. Keal, and W. Thiel, Chem. Phys. 349, 334 (2008).

[44] J. Pittner, H. Lischka, and M. Barbatti, Chem. Phys., doi:10.1016/j.chemphys.2008.10.013 (2008).

[45] J. C. Tully, J. Chem. Phys. 93, 1061 (1990).

[46] L. Sun, and W. L. Hase, in Reviews in Computational Chemistry, edited by K. B. Lipkowitz et al. (Wiley-VCH, New York, 2003), pp. 79.

[47] H. Lischka, R. Shepard, F. B. Brown, and I. Shavitt, Int. J. Quantum Chem. S.15, 91 (1981).

[48] H. Lischka, R. Shepard, R. M. Pitzer, I. Shavitt, M. Dallos, T. Müller, P. G. Szalay, M. Seth, G. S. Kedziora, S. Yabushita, and Z. Y. Zhang, PCCP 3, 664 (2001).

[49] H. Lischka, R. Shepard, I. Shavitt, R. M. Pitzer, M. Dallos, T. Mueller, P. G. Szalay, F. B. Brown, R. Ahlrichs, H. J. Boehm, A. Chang, D. C. Comeau, R. Gdanitz, H. Dachsel, C. Ehrhardt, M. Ernzerhof, P. Hoechtl, S. Irle, G. Kedziora, T. Kovar, V. Parasuk, M. J. M. Pepper, P. Scharf, H. Schiffer, M. Schindler, M. Schueler, M. Seth, E. A. Stahlberg, J.-G. Zhao, S. Yabushita, Z. Zhang, M. Barbatti, S. Matsika, M. Schuurmann, D. R. Yarkony, S. R. Brozell, E. V. Beck, and J.P. Blaudeau, COLUMBUS, an ab initio electronic structure program, release 5.9.1, www.univie.ac.at/columbus (2006).

[50] A. Bunge, J. Chem. Phys. 53, 20 (1970).

[51] T. H. Dunning, J. Chem. Phys. 90, 1007 (1989). 
[52] R. Shepard, H. Lischka, P. G. Szalay, T. Kovar, and M. Ernzerhof, J. Chem. Phys. 96, 2085 (1992).

[53] R. Shepard, in Modern Electronic Structure Theory, edited by D. R. Yarkony (World Scientific, Singapore, 1995), p. 345.

[54] H. Lischka, M. Dallos, and R. Shepard, Mol. Phys. 100, 1647 (2002).

[55] M. Dallos, H. Lischka, R. Shepard, D. R. Yarkony, and P. G. Szalay, Journal of Chemical Physics 120, 7330 (2004).

[56] H. Lischka, M. Dallos, P. G. Szalay, D. R. Yarkony, and R. Shepard, Journal of Chemical Physics 120, 7322 (2004).

[57] S. R. Langhoff, and E. R. Davidson, Int. J. Quantum Chem. 8, 61 (1974).

[58] P. J. Bruna, S. D. Peyerimhoff, and R. J. Buenker, Chem. Phys. Lett. 72, 278 (1980).

[59] V. Bonacic-Koutecky, and R. Mitric, Chem. Rev. 105, 11 (2005).

[60] R. Mitric, V. Bonacic-Koutecky, J. Pittner, and H. Lischka, J. Chem. Phys. 125 (2006).

[61] W. C. Swope, H. C. Andersen, P. H. Berens, and K. R. Wilson, J. Chem. Phys. 76, 637 (1982).

[62] G. Granucci, and M. Persico, J. Chem. Phys. 126, 134114 (2007).

[63] D. Cremer, and J. A. Pople, J. Am. Chem. Soc. 97, 1354 (1975).

[64] T. Helgaker, H. J. A. Jensen, P. Jørgensen, J. Olsen, K. Ruud, H. Ågren, T. Andersen, K. L. Bak, V. Bakken, O. Christiansen, P. Dahle, E. K. Dalskov, T. Enevoldsen, H. Heiberg, H. Hettema, D. Jonsson, S. Kirpekar, R. Kobayashi, H. Koch, K. V. Mikkelsen, P. Norman, M. J. Packer, T. Saue, P. R. Taylor, and O. Vahtras, DALTON, an ab initio electronic structure program, Release 1.0 (1997).

[65] M. Barbatti, G. Granucci, M. Ruckenbauer, M. Persico, and H. Lischka, NEWTON-X: a package for Newtonian dynamics close to the crossing seam, www.univie.ac.at/newtonx (2007). 


\section{Tables}

Table 1 - Vertical excitation energies of selected singlet states of pyrrole.

\begin{tabular}{llcccccc}
\hline State & MRCI $^{\mathrm{a}}$ & ${\text { MRCI }+\mathrm{Q}^{\mathrm{b}}}^{\mathrm{T}}$ & TDDFT $^{\mathrm{c}}$ & $\mathrm{CASPT}^{2}$ & $\mathrm{CC}^{\mathrm{g}}$ & Exp \\
\hline $\mathrm{S}_{0}$ & ${ }^{\mathrm{I}} \mathrm{A}_{1}$ & 0.00 & 0.00 & 0.00 & 0.00 & 0.00 & \\
$\pi 3 \mathrm{~s}$ & ${ }^{1} \mathrm{~A}_{2}$ & 5.22 & 5.09 & 5.05 & $5.22^{\mathrm{d}} / 5.22^{\mathrm{e}}$ & 5.10 & $5.08^{\mathrm{h}} / 5.22^{\mathrm{i}}$ \\
$\pi 3 \mathrm{~s}$ & ${ }^{1} \mathrm{~B}_{1}$ & 6.21 & 5.86 & 5.88 & $5.85^{\mathrm{f}} / 5.92^{\mathrm{e}}$ & 5.99 & $6.22^{\mathrm{h}}$ \\
$\pi \pi^{*}$ & ${ }^{1} \mathrm{~A}_{1}$ & 6.55 & 6.39 & 6.29 & $5.82^{\mathrm{d}} / 5.98^{\mathrm{e}}$ & 6.37 & \\
$\pi \pi^{*}$ & ${ }^{1} \mathrm{~B}_{2}$ & 6.65 & 6.78 & 6.45 & $5.87^{\mathrm{d}} / 5.95^{\mathrm{e}}$ & 6.63 & $5.92^{\mathrm{h}} / 6.2-6.4^{\mathrm{i}}$ \\
\hline
\end{tabular}

${ }^{a}$ Present results, MR-CISD/SA-5-CASSCF(4,5)/BS

${ }^{\mathrm{b}} \mathrm{MR}-\mathrm{CISD}+\mathrm{Q} / \mathrm{SA}-5-\mathrm{CAS}(6,5)+\mathrm{AUX}(1) / \mathrm{d}$-aug-cc-pVDZ, Reference [27].

${ }^{\mathrm{c}}$ Reference [4].

${ }^{\mathrm{d}}$ Reference [6].

${ }^{\mathrm{e}}$ Reference [8].

${ }^{\mathrm{f}}$ Reference [1].

${ }^{\mathrm{g}}$ Reference [3].

${ }^{\mathrm{h}}$ Assigments given in Reference [9].

${ }^{\mathrm{i}}$ Assigments given in Reference [3]. 
Table 2- Energy of pyrrole MXSs (in eV) relative to the minimum in the ground state.

\begin{tabular}{llllll}
\hline MXS & MRCI $^{\mathrm{a}}$ & MRCI+Q $^{\mathrm{a}}$ & MRCI & MRCI+Q & MXS features \\
\hline$\pi \pi^{*} / \mathrm{S}_{0}\left(\mathrm{E}_{1}\right)$ & 4.95 & 4.86 & $4.89^{\mathrm{b}}$ & $4.93^{\mathrm{b}}$ & ring puckering, Fig. 1a \\
$\pi \sigma_{\mathrm{NH}} * / \mathrm{S}_{0}$ & 4.45 & 4.26 & $4.41^{\mathrm{c}}$ & $4.44^{\mathrm{c}}$ & NH stretching, Fig. 1b \\
$\pi \sigma_{\mathrm{NC}} * \mathrm{~S}_{0}$ & 4.11 & 3.86 & - & - & ring opening, Fig. 1c \\
\hline esent results, MR-CISD/SA-5-CASSCF(4,5)/BS & & \\
R-CISD(Q)/SA-3-CAS(6,5)/6-31G(d), Reference [27]. & \\
R-CISD(Q)/SA-3-CAS(6,6)/6-31G(d), Reference [27].
\end{tabular}

${ }^{b}$ MR-CISD(Q)/SA-3-CAS(6,5)/6-31G(d), Reference [27]

${ }^{c}$ MR-CISD(Q)/SA-3-CAS(6,6)/6-31G(d), Reference [27]. 


\section{Figure Captions}

Fig. 1. Structures and selected geometric parameters for pyrrole MXSs obtained at the MRCI level. Distances are given in $\AA$ and dihedral angles in degrees. The number in brackets correspond to the benchmark MRCI value from Ref. [27].

Fig. 2. Potential energy curves calculated at the MRCI level along a) the rigid NH-stretching coordinate and along the LIIC path from the ground state minimum to b) the ring-puckered MXS and to c) the ring-opened MXS.

Fig. 3. Average adiabatic populations of trajectories for each state as a function of time after initial photoexcitation of pyrrole into the $\mathrm{S}_{4}$ state.

Fig. 4. Description and statistics of trajectory deactivation mechanisms.

Fig. 5. CN (top) and NH (bottom) distance variations as a function of time for all trajectories. The NH distance of $10 \AA$ was used as a cut-off value (see text).

Fig. 6. Analysis of the trajectories showing NH dissociation. (a) Initial time of the dissociation, taking $2 \AA$ for the $\mathrm{NH}$ bond as reference value. (b) Hydrogen kinetic energy. (c) NH distance for all trajectories at the time of the $\mathrm{S}_{1} \rightarrow \mathrm{S}_{0}$ hopping.

Fig. 7. Distribution of conformations in the Cremer-Pople Q- $\phi$ space for trajectories following ringdeformation mechanisms. Full dots: conformations at the hopping time. Open dots: conformations with $\mathrm{S}_{1}-\mathrm{S}_{0}$ energy gaps smaller than $0.5 \mathrm{eV}$. Cross: ring puckered MXS. 
a) Ring-puckered MXS c) Ring-opened MXS
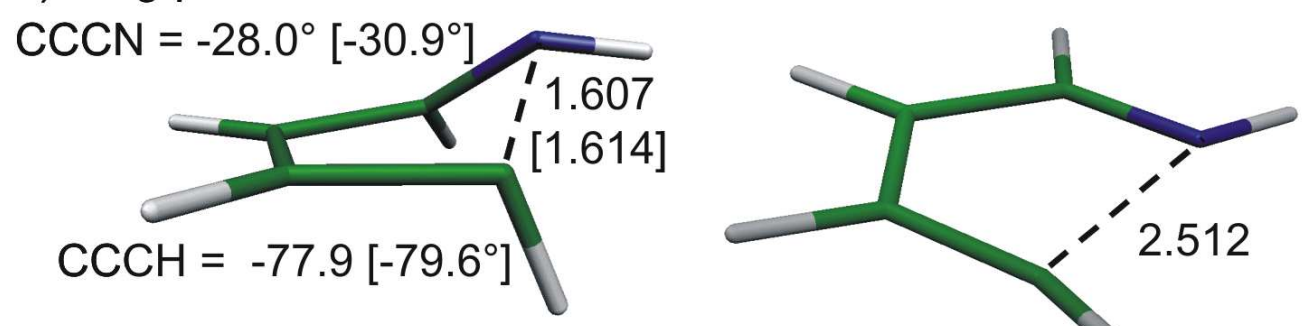

b) NH-dissociation MXS

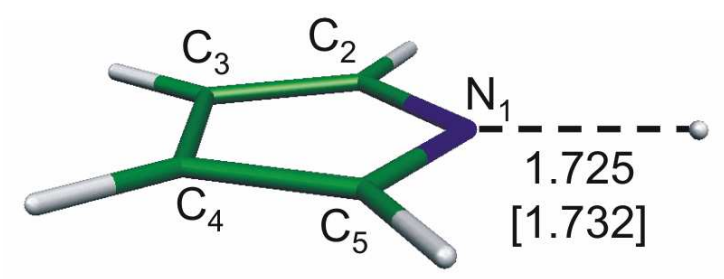



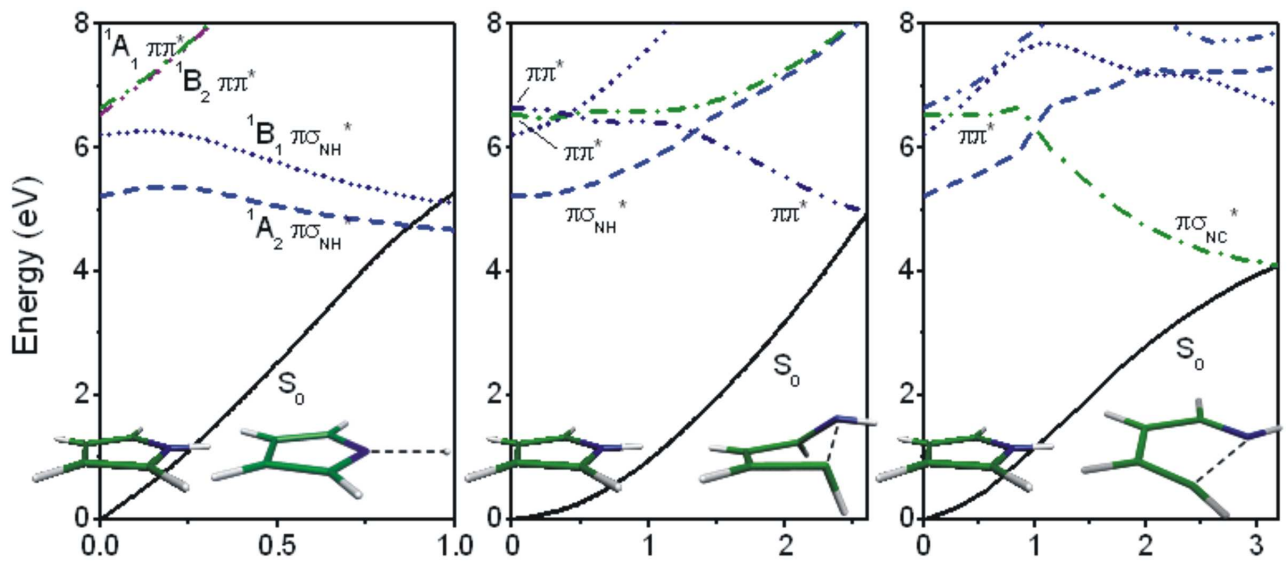

Mass-weighted distance $\left(\AA . . a m u^{1 / 2}\right)$ 


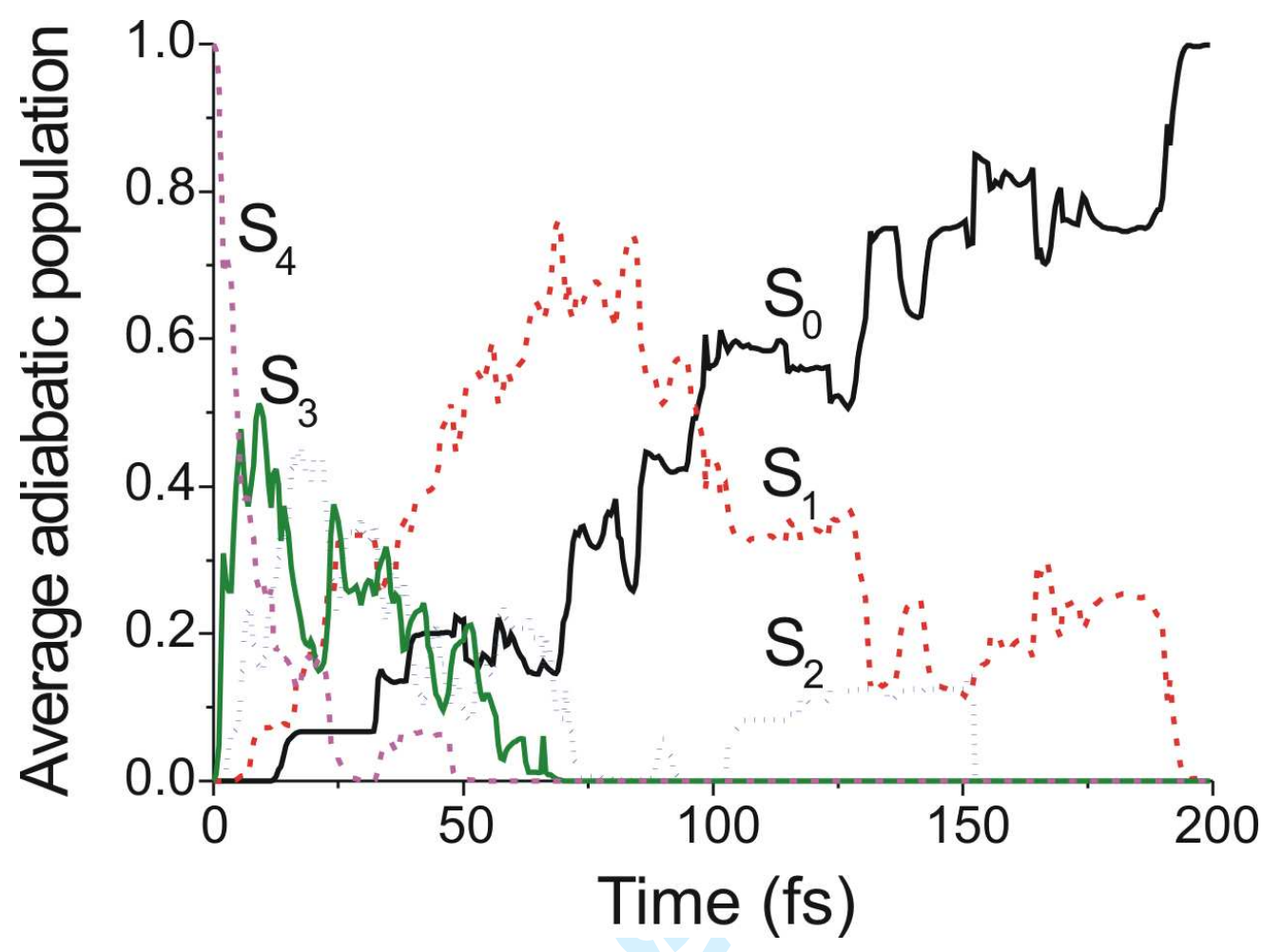




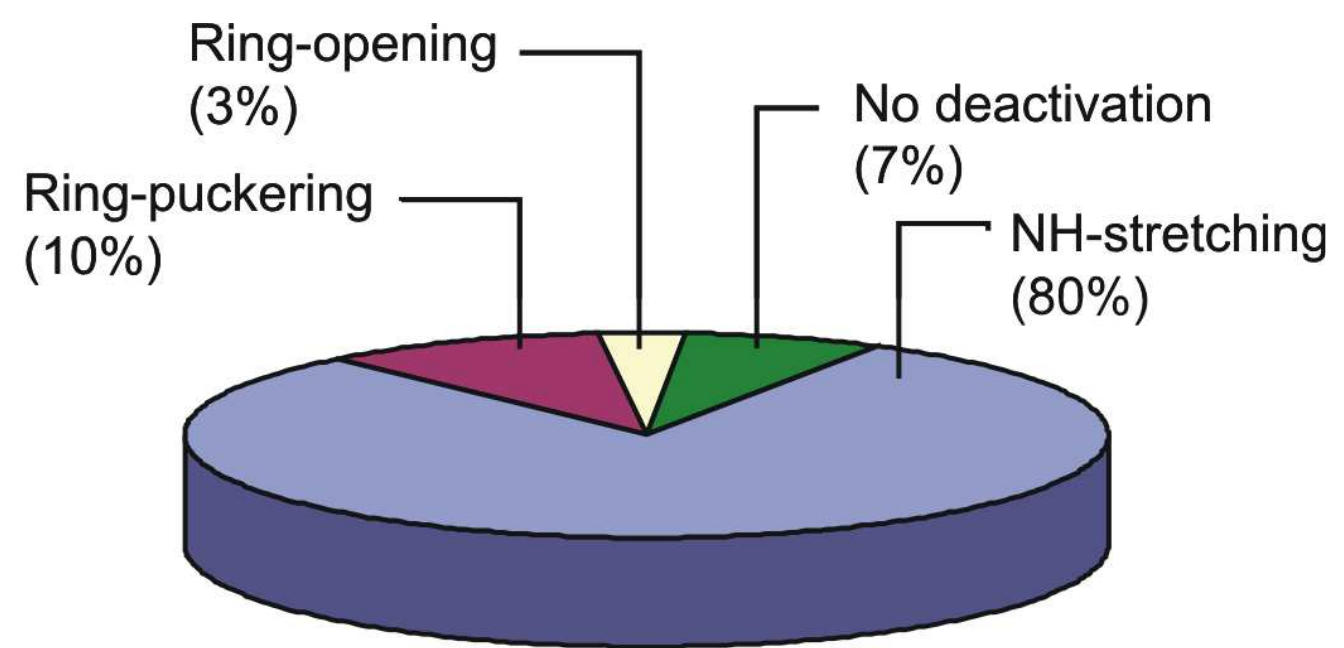




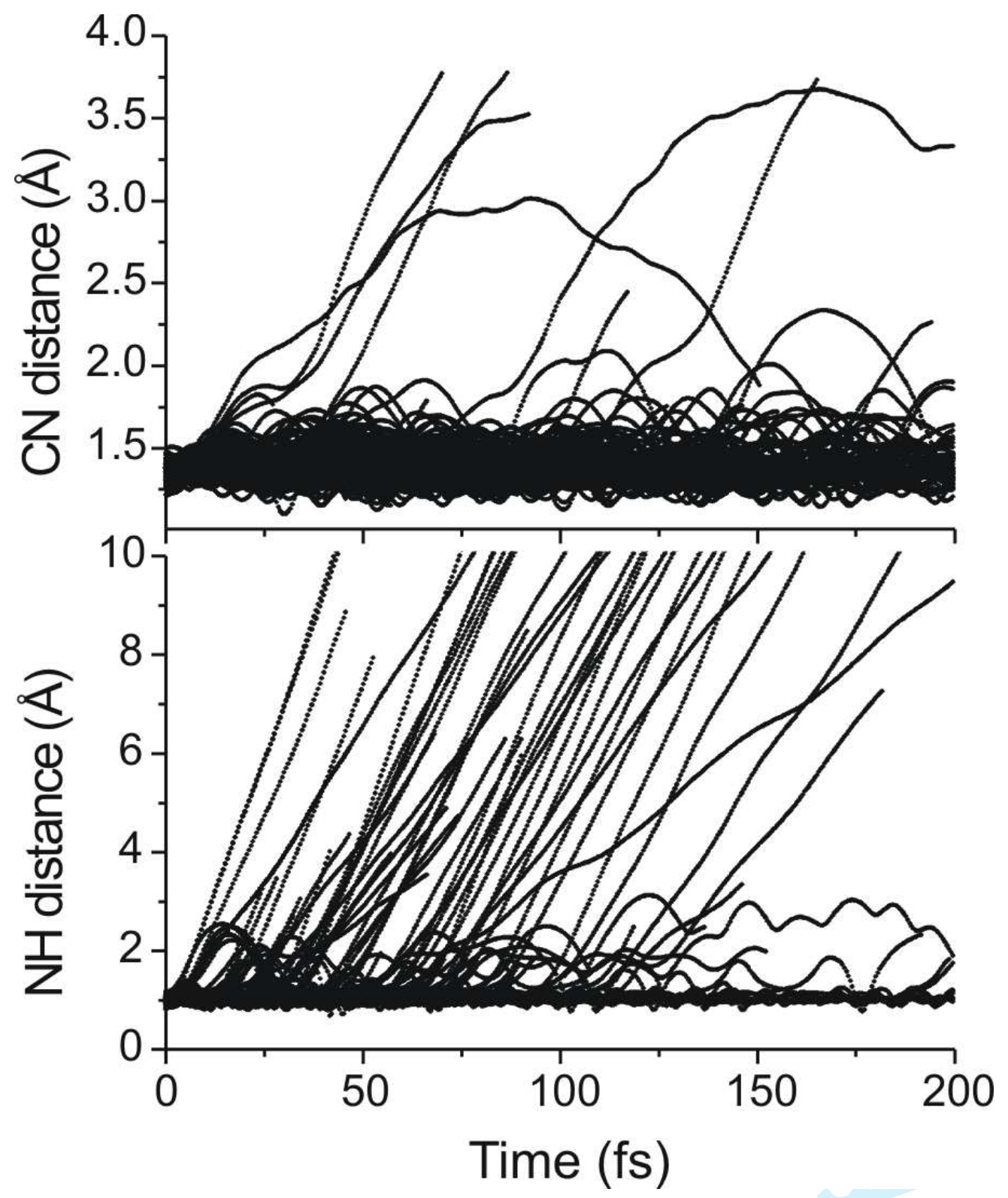




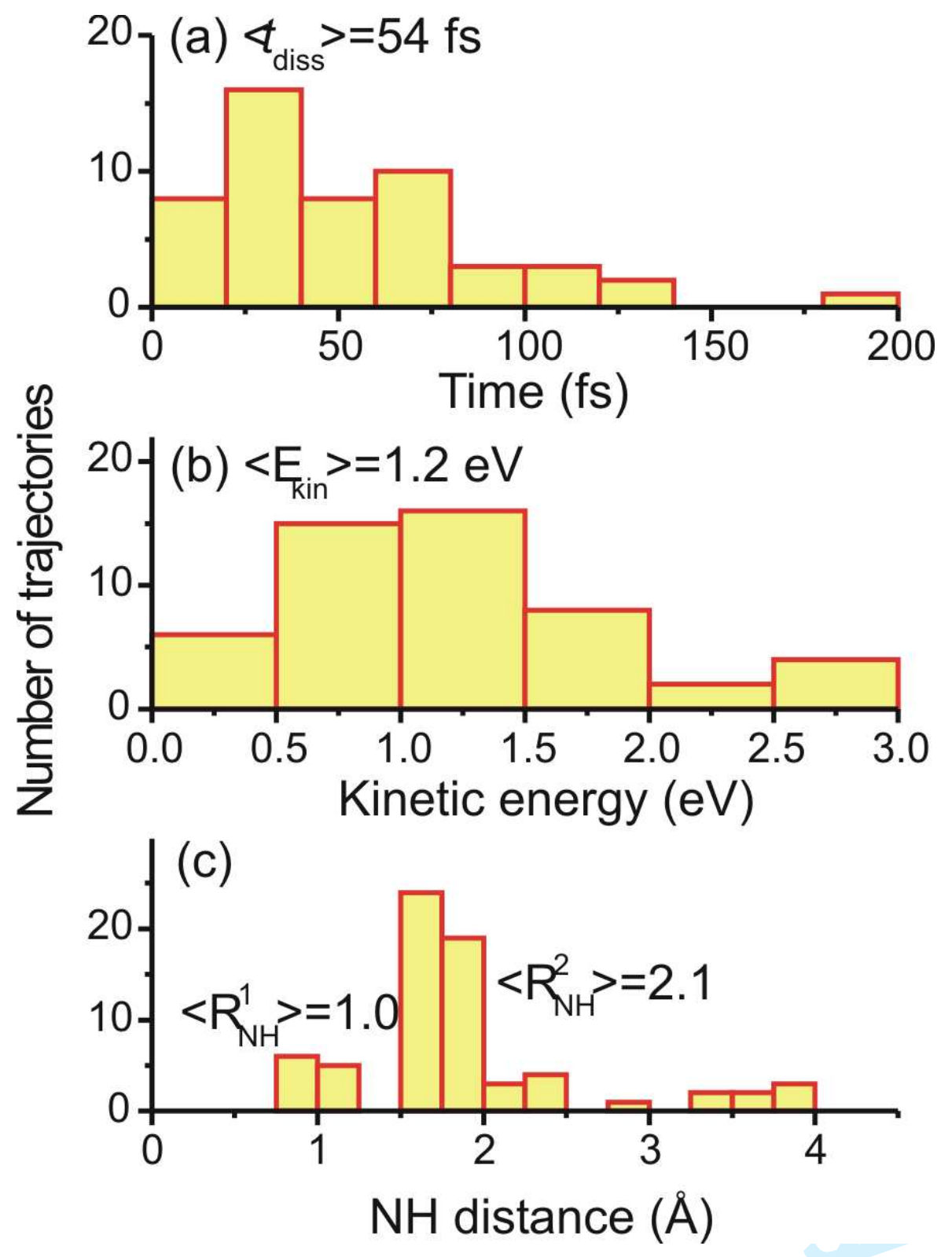

URL: http://mc.manuscriptcentral.com/tandf/tmph 


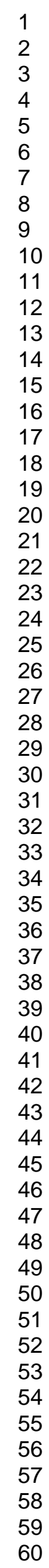

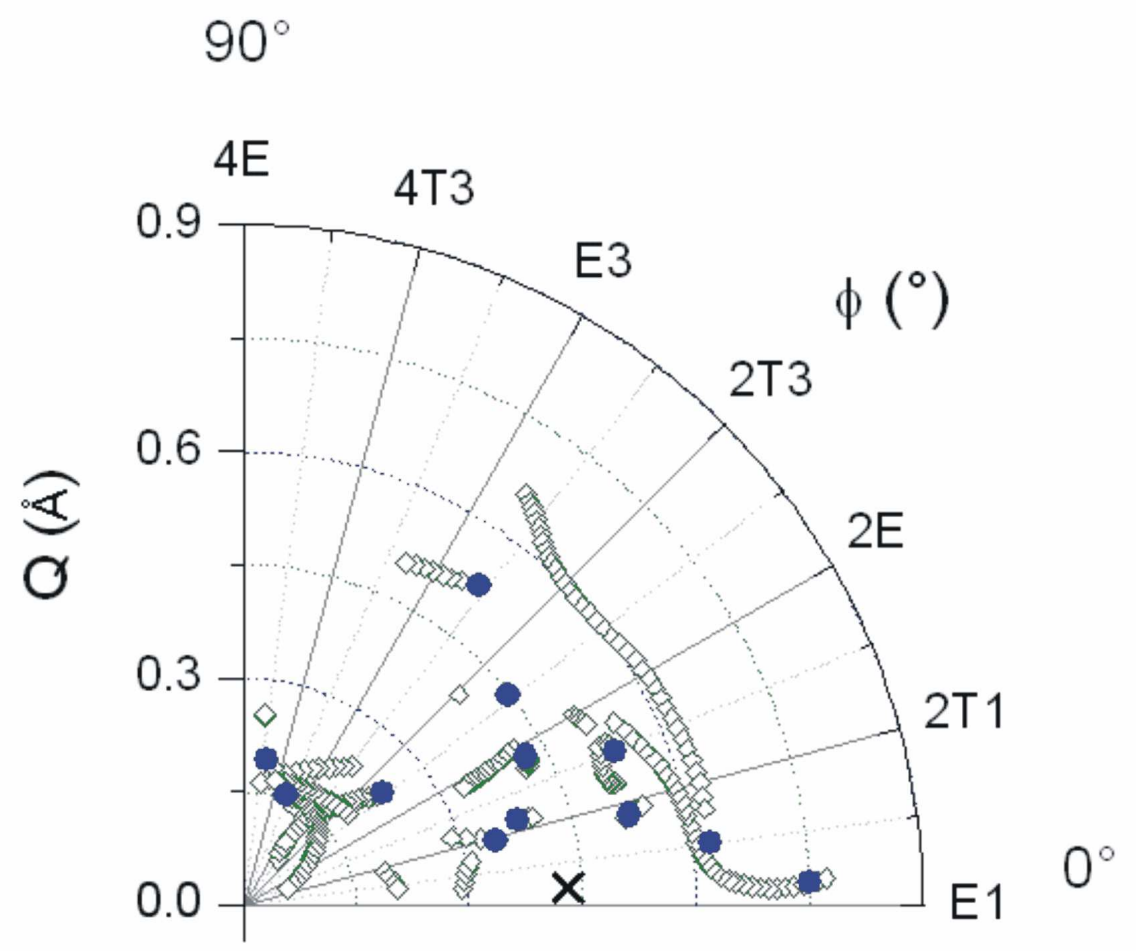

URL: http://mc.manuscriptcentral.com/tandf/tmph 


\title{
Excited-state non-adiabatic dynamics simulations of pyrrole
}

\author{
Mario Vazdar, ${ }^{\mathrm{a}}$ Mirjana Eckert-Maksić, ${ }^{\mathrm{a}^{*}}$ Mario Barbatti, ${ }^{\mathrm{b}^{*}}$ Hans Lischka ${ }^{\mathrm{b}^{*}}$ \\ ${ }^{a}$ Laboratory for Physical-Organic Chemistry - Division of Organic Chemistry and \\ Biochemistry. Rudjer Bošković Institute, 10002 Zagreb, Croatia; ${ }^{b}$ Institute for Theoretical \\ Chemistry - University of Vienna, Waehringerstrasse 17, A 1090 Vienna, Austria.
}

\begin{abstract}
Non-adiabatic on-the-fly-dynamics simulations of the photodynamics of pyrrole were performed at multireference configuration interaction level involving five electronic states with a simulation time of 200 fs. The analysis of the time dependence of the average state occupations shows that the deactivation of pyrrole to the electronic ground state takes place in about 140 fs. This deactivation time agrees very well with the experimentally measured time constant of $110 \mathrm{fs}$ for the formation of fast hydrogen atoms. After excitation into the $\mathrm{S}_{4}$ state, $80 \%$ of the trajectories followed the NH-stretching mechanism giving rise to a population of fast $\mathrm{H}$ atoms. The computed average kinetic energy is in good accord with the experimentally observed average kinetic energy of the fast hydrogen atoms. It is found that $10 \%$ of trajectories followed the ring-puckering mechanism and $3 \%$ followed the ring-opening mechanism. This latter mechanism was characterized in pyrrole for the first time and involves the conical intersection of lowest energy of this molecule.
\end{abstract}

Keywords: non-adiabatic dynamics; conical intersection; photochemistry; pyrrole

* Corresponding authors: H. Lischka (hans.lischka@univie.ac.at), M. Barbatti (mario.barbatti@univie.ac.at) and M. Eckert-Maksić (mmaksic@emma.irb.hr) 


\section{Introduction}

Pyrrole is one of the simplest biologically relevant heteroaromatic compounds. For this reason, its electronic states have been intensively studied both experimentally and theoretically during the last decades with the emphasis on its UV spectrum [1-10] and its photodynamics [11-24]. In particular, it is known that the deactivation of UV-excited pyrrole to the ground state occurs at a very short (femtosecond) time scale [19] with low luminescence quantum yields [25], indicating the dominance of internal conversion processes. Sobolewski and coworkers [13] have proposed that the deactivation of pyrrole and related heteroatomic compounds occurs via the $\mathrm{NH}$-stretching mechanism along a ${ }^{1} \pi \sigma_{\mathrm{NH}} *$ repulsive state. This mechanism, which has been examined in detail $[15,16,22,23]$ by means of wave packet dynamics simulations, can fully explain the presence of fast $\mathrm{H}$ atoms in the photofragmentation spectra [26]. Nevertheless, the mechanism responsible for the formation of slow $\mathrm{H}$ atoms and of other experimentally observed fragments such as $\mathrm{HCN}$ and $\mathrm{CNH}_{2}[11$, $14,17,19,20]$ is still subject of considerable debate $[15,19,26-28]$.

Recently, we have suggested [27] that non-adiabatic deactivation of pyrrole may also proceed via a ring-puckering mechanism. This second kind of mechanism could not only be the source of heavy fragments, but also partially explain the slow H atoms [29]. Also recently, a third deactivation mechanism that can be relevant for pyrrole was identified in thiophene [30], furan [31], imidazole [32], and in the imidazole group of adenine [33]. In this mechanism the deactivation of five-membered rings proceeds by a planar ring-opening deformation. This process was observed to occur in a minor fraction of trajectories during dynamics simulations of adenine [34]. Based on these findings, we have currently attempted and succeeded to locate this type of mechanism in pyrrole, too. 
Profant et al. [35] and Poterya et al. [28] have experimentally investigated the photolysis of pyrrole clusters. In addition, they have also performed theoretical calculations on the isolated pyrrole and on pyrrole complexes [28]. They have found that in presence of solvent molecules the $\mathrm{NH}$ stretching mechanism is inhibited, which results in a strong reduction of the fast $\mathrm{H}$ atom elimination process while keeping the slow $\mathrm{H}$ atom elimination. These are important results that on one hand once more confirm the role of the NH-stretching mechanism for the fast $\mathrm{H}$ atoms formation and on the other hand indicate that ring deformation mechanisms should be involved in the slow $\mathrm{H}$ atoms formation.

The strong dependence of different fragment yields on the excitation energy [26] indicates that the individual mechanisms are in mutual competition and can play different roles depending on the initial conditions. Excited-state energy surfaces have been investigated in detail under special consideration of crossings between different energy surfaces as already mentioned above $[1,18,23,27,28,36]$ and reaction paths have been constructed subsequently. This information led to substantial progress in the understanding of the photochemical processes in pyrrole. However, it turned out to be very difficult to estimate the importance of individual intersections and related reaction pathways. In order to better understand how these mechanisms are activated, it is desirable to perform dynamics simulations. Such simulations exhibit a substantial complexity. For instance, as for selecting the proper quantum chemical methods, it needs to be taken into account that: first, the nonadiabatic dynamics of pyrrole involves multiple excited states showing often multireference character and, secondly, that it is essentially impossible to identify just a few important internal degrees of freedom by which the photochemical reaction mechanism can be described. Therefore, an essential condition is the usage of the full set of nuclear coordinates. These are usual requirements to be met e.g. in simulations of organic chromophores exhibiting high density of excited states $[34,37,38]$. One convenient way to satisfy especially 
the second condition is to use mixed-quantum classical dynamics methods [39-44]. In this work surface hopping dynamics is performed using the fewest-switches algorithm of Tully [45]. The advantage of this approach is that it allows the application of an "on-the-fly" strategy $[42,43,46]$ where a pre-selection of certain internal degrees of freedom and any fitting of pre-computed potential energy points is avoided by computing at each time step the energies, the complete energy gradient and non-adiabatic coupling terms required for the integration of Newton's equations of motion and the time-dependent Schrödinger equation. This on-the-fly strategy is computationally very expensive and requires analytical energy gradients and non-adiabatic coupling vectors for computational efficiency. Due to the stringent computational requirements most of the photodynamical simulations have been performed so far at the relatively cost-effective complete active space self consistent field level (CASSCF). Since in this case dynamical electron correlation effects are mostly neglected, the relative balance of electronic states of different character can be strongly violated. It should be stressed that the non-adiabatic dynamics simulations presented here were carried out at a significantly higher level using the MR-CISD method including five electronic states. This represents the state-of-the-art approach for this kind of simulations, which has not been documented before for molecules of the size of pyrrole to the best of our knowledge. The present calculations have been made possible by use of the analytic gradient features of the program package COLUMBUS [47-49] as it will be described below.

\section{Computational details}

Multireference configuration interaction (MRCI) and complete active space self-consistent field (CASSCF) calculations were performed for pyrrole. The CAS space was comprised of four $\pi$ electrons in five orbitals (two $\pi$ orbitals, two $\pi^{*}$ orbitals and one Rydberg $3 \mathrm{~s}$ orbital). This space will be conventionally designated as $\operatorname{CAS}(4,5)$ in the text. State averaging was 
performed over five singlet states with equal weights (ground state, two valence $\pi \pi^{*}$ states and two Rydberg $\pi 3 \mathrm{~s}$ states), which will be denoted as SA-5. MRCI calculations were performed based on the orbitals computed by the $\operatorname{SA}-5-\operatorname{CASSCF}(4,5)$ wave function. The reference configurations for the MRCI were constructed within the CAS(4,5) by allowing single and double excitations from the two $\pi$ orbitals into the two $\pi^{*}$ orbitals and the Rydberg 3s orbital. The final configuration space was constructed by allowing all single and double excitations from the reference configurations into the virtual orbital space (MR-CISD). All core electrons and the lowest eight additional orbitals were frozen in the MRCI calculations and the interacting space restriction [50] was applied. The basis set was composed of aug'-ccpVDZ type [51] on the nitrogen and carbon atoms (the prime indicates that d-aug functions were removed). On the hydrogen atom connected to nitrogen, the cc-pVDZ basis set was used, whereas for the remaining hydrogen atoms the cc'-pVDZ basis set was used (the prime signifies that p-functions were deleted). This hybrid basis set will be denoted as BS.

The MRCI approach and the basis set were selected by balancing the accuracy requirements of the calculations of four excited states of different character (see Table 1) and the need for computational efficiency, since an on-the-fly approach requires several tens of thousands of individual MRCI calculations to be carried out. Therefore, before starting the dynamics simulations an extensive set of calculations had been performed, including the Franck-Condon region, the seam of conical intersections, and reaction pathways. For the determination of minima on the crossing seam (MXS), starting geometries were selected from our previous MRCI calculations on pyrrole [27] and were reoptimized with the abovedescribed MRCI method. Reaction paths for the two ring-deformation processes were constructed by the method of linear interpolation of internal coordinates (LIIC) between the ground-state geometry and the corresponding ring-deformed conical intersections. The reaction path for the $\mathrm{NH}$-stretching process was constructed by rigidly stretching the $\mathrm{NH}$ 
distance in steps of $0.2 \AA$ starting from the ground state equilibrium structure up to a $\mathrm{NH}$ distance of $2.6 \AA$.

All energy calculations and MXS optimizations were performed by using analytical gradient and non-adiabatic coupling procedures described in References [52-56]. For vertical excitation energy calculations, the Davidson correction (+Q) $[53,57,58]$ was used in order to describe higher order excitation effects. For the $\mathrm{C}_{2 \mathrm{v}}$ labeling of the states, the $x$ axis was assumed to be oriented perpendicular to the ring plane.

Mixed quantum-classical dynamics calculations were performed for pyrrole by using an on-the-fly approach $[42,43,46,59,60]$. Energies, gradients, and non-adiabatic coupling vectors were computed at each time step at the MR-CISD/SA-5-CASSCF(4,5)/BS level of theory. The nuclear motion was represented by classical trajectories computed by numerical integration of Newton's equations by the velocity-Verlet algorithm [61]. Non-adiabatic effects were taken into account by means of the surface hopping approach [45]. Time-dependent adiabatic populations were corrected for decoherence effects [62] ( $\alpha=0.1$ hartree) and used to calculate surface hopping probabilities in accordance to the Tully's fewest switches approach [45]. In order to alleviate the computational costs, no coupling vectors were calculated between non-consecutive states [44]. In total, 90 trajectories were computed. The initial Cartesian coordinates and momenta were selected from a quantum harmonic oscillator (Wigner) distribution in the ground state. The trajectories were started in the $\mathrm{S}_{4}$ state at these geometries. This procedure gave rise to a composition of $60 \%$ of trajectories initially in the $\pi \pi^{*}$ states and $40 \%$ in the $\pi 3 \mathrm{~s} / \pi \sigma_{\mathrm{NH}} *$ states. The minimum excitation energy was $6.36 \mathrm{eV}$ while the average was $6.76 \mathrm{eV}$ with a standard deviation of $0.26 \mathrm{eV}$. The trajectories were then propagated for a maximum time of $200 \mathrm{fs}$ with a time step of $0.5 \mathrm{fs}$.

The structures of the puckered geometries were described in terms of the CremerPople parameters $\mathrm{Q}$ and $\phi[63]$. While the parameter $\mathrm{Q}$ measures the extent of puckering $(\mathrm{Q}=$ 
$0 \AA$ indicates a planar structure), the parameter $\phi$ describes the kind of puckering. For 5mebered rings, there are only few kinds of puckered conformations available: envelope conformations with atom $k$ above $\left({ }^{k} \mathrm{E}\right)$ or below $\left(\mathrm{E}_{k}\right)$ the ring plane and twisted conformations with atom $k$ above the ring plane and atom $k$-1 below the ring plane $\left({ }^{k} \mathrm{~T}_{k-1}\right)$. Because of the pyrrole symmetry, $\phi$ can be reduced to the $0^{\circ}-90^{\circ}$ range by projecting all values on this quadrant.

All CASSCF and MR-CISD+Q calculations were performed with the COLUMBUS [47-49] program package. The atomic orbital (AO) integrals and AO gradient integrals have been calculated with program modules taken from DALTON [64]. The dynamic simulations were carried out using the NEWTON-X program [42, 65] with an interface to the COLUMBUS program package.

Table 1. (around here)

\section{Analysis of the energy surfaces}

In order to investigate the reliability of the MRCI method used in the dynamics study, we have performed a series of tests and comparisons with other previously published results. Specifically, we have compared vertical excitation energies, reaction paths, and MXS structures with results obtained with methods of higher level of theory.

\subsection{Vertical excitation energies}

The theoretical computation of vertical excitation energies of pyrrole and the assignment of the experimental UV spectrum have been a matter of discussion for a long period of time [1, $3,4,8,9]$. The currently calculated values are compared to other available theoretical and experimental results in Table 1. The comparison reveals that vertical excitation energies computed by the MR-CISD/SA-5-CASSCF(4,5)/BS method are in good accordance with 
Fig. 1 (around here)

\subsection{Conical intersections}

In Fig. 1 the MXS structures between ground state and the $S_{1}$ state are presented. The comparison of selected geometrical parameters for the ring-puckered (Fig. 1a) and the NHstretched (Fig. 1b) MXS structures reveals that they are in very good agreement with the benchmark MRCI values [27].

In Fig. 1a, the MXS between the valence $\pi \pi^{*}$ state and the ground state shows an out-ofplane deformation with strong stretching of one of the $\mathrm{CN}$ bonds. We shall refer to this conical intersection as the ring-puckered MXS. The values of dihedral $\mathrm{CCCN}$ and $\mathrm{CCCH}$ dihedral angles are very close to the benchmark ones, being only by ca. $2^{\circ}$ smaller. The length of the broken $\mathrm{CN}$ bond is $1.607 \AA$, thus being $0.007 \AA$ shorter than the value obtained with the benchmark method. In Fig. 1b, the NH-stretched MXS is shown. It arises from the crossing 
between the ground state and the lowest $\pi \sigma_{\mathrm{NH}}{ }^{*}$ state. In comparison to the benchmark MRCI value, the NH distance using the current method is shorter by $0.007 \AA$.

As mentioned in the Introduction, based on previous findings for other five-membered heteroaromatic molecules [30, 31, 33], we have searched for a planar ring-opened MXS in pyrrole as well. The optimized structure, obtained at the MRCI level of theory, is presented in Fig. 1c. It should be pointed out that the MXS is planar and that the CN distance is $2.512 \AA$, which is by about $0.9 \AA$ longer than the $\mathrm{CN}$ distance observed in the ring puckered MXS (Fig. 1a). It is important to note that the ring-opened MXS is the lowest energy conical intersection identified in pyrrole so far and it arises from the crossing between the $\pi \sigma_{\mathrm{NC}}{ }^{*}$ state and the ground state.

\section{Table 2. (around here)}

Although the similarity of geometrical parameters suggests that the selected MRCISD/SA-5-CASSCF(4,5)/BS level of theory is adequate, it is also of importance to compare the energies of the MXSs. MRCI and MRCI+Q energy values of pyrrole MXSs obtained by the MR-CISD(Q)/SA-5-CASSCF(4,5)/BS and benchmark MRCI values [27] are summarized in Table 2. The analysis of presented data shows that the energies of the MXSs are in very good agreement with the benchmark ones. The comparison among results reveals that the selected MRCI method is well suited for the description of both ring-puckering and NHstretching mechanisms. In particular, the current MRCI and MRCI+Q energies of the ringpuckered MXS are by $0.06 \mathrm{eV}$ higher and $0.07 \mathrm{eV}$ lower than the benchmark MRCI and MRCI+Q values, respectively. For the NH-stretched MXS, the MRCI energy is by $0.04 \mathrm{eV}$ higher, whereas the MRCI+Q value is by $0.18 \mathrm{eV}$ lower than the benchmark values. 


\subsection{Reaction paths}

We have computed the reaction pathways between the ground state minimum and the three MXSs described in the previous section using the MR-CISD/SA-5-CASSCF(4,5)/BS level of theory. The resulting potential energy curves are shown in Fig. 2.

Fig. 2 (around here)

Comparison with the results obtained with the MR-CISD+Q/SA-5-
CAS(6,5)+AUX(1)/d-aug-cc-pVDZ method (Fig. 2 in [27]) reveals that NH-stretching potential energy curves (Fig. 2a) agree very well for the ground state $S_{0}$ and the two Rydberg ${ }^{1} \mathrm{~A}_{2}$ and ${ }^{1} \mathrm{~B}_{1}$ states for the whole range of $\mathrm{NH}$ distances. The main difference is that the crossing between the lowest Rydberg state and the ground state occurs at around $1.9 \AA$ in the present work instead of $2.1 \AA$ found in our earlier study [27]. The other features of the potential energy curves exhibit the same behavior as observed earlier. Specifically, the lowest two Rydberg states show small energy barriers $\left(0.24 \mathrm{eV}\right.$ for the ${ }^{1} \mathrm{~A}_{2}$ state and 0.12 for the ${ }^{1} \mathrm{~B}_{1}$ state) at the $\mathrm{NH}$ distance of $1.2 \AA$ necessary to transform the $\pi 3 \mathrm{~s}$ orbital into the $\pi \sigma_{\mathrm{NH}}{ }^{*}$ state as expected for a stretching of the $\mathrm{NH}$ bond. It should also be pointed out that the two valence ${ }^{1} \mathrm{~A}_{1}$ and ${ }^{1} \mathrm{~B}_{2}$ states show the same energy profile until the NH distance of $1.8 \AA$. After that, an intrusion of higher excited states occurs (not shown), which is presumably a direct consequence of the CAS $(4,5)$ active space. However, in the NH stretching mechanism, the deactivation occurs via conical intersections among Rydberg states and the ground state and the differences in the valence states for large NH distances are of minor importance.

Fig. $2 b$ shows that the LIIC path of the ring-puckering mechanism in the $\pi \pi^{*}$ state occurs without barrier. Indeed, it is clearly seen that the lowest $\pi \pi^{*}$ state is diabatically connected to the ground state, which may make it especially efficient for the internal conversion. The same result was observed in our previous study [27] thus providing additional 
support for using the applied method. In the case of the ring-opening mechanism Fig. 2c shows that the initially excited $\pi \pi^{*}$ states can deactivate without barrier along this pathway. The character of the state should, however, change into $\pi \sigma_{\mathrm{NC}} *$ in order to lead to the crossing with the ground state.

Apart from the fact that the $\mathrm{NH}$-stretching mechanism should dominate at low excitation energies, it is difficult to draw general conclusions about the efficiency of each mechanism based on the reaction paths alone in a clear cut way. When the excitation leads into the spectral region of the $\pi \pi^{*}$ state all mechanisms are energetically possible. In favor of the $\mathrm{NH}$-stretching mechanism is the fact that it requires the smallest deformations from the Franck-Condon region in terms of mass-weighted distances (see Fig. 2). On the other hand, it also requires the diabatic transformation from the $\pi \pi^{*}$ state into the $\pi \sigma_{\mathrm{NH}}{ }^{*}$ state, which depends upon the activation of out-of-plane modes $[18,27]$. The ring-opening mechanism involves the lowest energy conical intersection, but it requires the largest deformations from the Franck-Condon region and diabatic changes in the wave function at the same time. Finally, the ring-puckering mechanism, as already mentioned, can directly proceed through a diabatic connection. However, it involves the highest energy portions of the seam of conical intersections.

\section{Dynamics simulations of pyrrole}

The non-adiabatic excited state dynamics of pyrrole was started from the $\mathrm{S}_{4}$ state thus making all pathways discussed in the previous section energetically available. The resulting average adiabatic populations of the ground and excited states as a function of time are presented in Fig. 3. Their analysis shows that the $S_{4}$ state transfers its population to the $S_{3}$ state in the first 10 fs. After ca. 50 fs, the $S_{4}$ state is almost completely depopulated. The populations of $S_{3}$ and $\mathrm{S}_{2}$ states reach a maximum at $10 \mathrm{fs}$ and $20 \mathrm{fs}$, respectively. At about $75 \mathrm{fs}$, these states are 
already depopulated. The $S_{2}$ state shows a repopulation between 100 and 150 fs. The population of the $S_{1}$ state increases reaching a maximum at 75 fs. At 100 fs, the $S_{1}$ and $S_{0}$ states have approximately the same population. Between $100 \mathrm{fs}$ and $200 \mathrm{fs}$, the simulation is basically reduced to the $S_{1} / S_{0}$ two-state dynamics, with the complete population transferred to the ground state at about $200 \mathrm{fs}$.

Fig. 3 (around here)

The $S_{1}$ population shows a consecutive two-step first order decay type of behavior. By fitting the $S_{1}$ population curve with the function

$$
f(t)=\frac{\tau_{2}}{\tau_{2}-\tau_{1}}\left[\exp \left(-\frac{t}{\tau_{1}}\right)-\exp \left(-\frac{t}{\tau_{2}}\right)\right]
$$

two time constants $\tau_{1}=44 \pm 2$ fs and $\tau_{2}=80 \pm 2$ fs are obtained. Here, $\tau_{1}$ measures the population of $S_{1}$ from the collection of states $S_{4}$ to $S_{2}$ and $\tau_{2}$ describes the depopulation $\mathrm{S}_{1} \rightarrow \mathrm{S}_{0}$. The approximate time constant for the overall population of the ground state can be obtained by fitting the $S_{0}$ population with the function

$$
f(t)=1-\exp \left(-\frac{t}{\tau_{0}}\right)
$$

which gives $\tau_{0}=139 \pm 2$ fs. Note that in these three time constants the error bars denote the uncertainty of the fitting procedure and not of the process itself, which certainly is larger than a few femtoseconds.

In Fig. 4 a summary of the results of the dynamics simulation in terms of the fraction of trajectories following each of the three mechanisms is given. The NH-stretching is the main mechanism after excitation of pyrrole to the $\mathrm{S}_{4}$ state. This mechanism occurs in $80 \%$ of the trajectories. Other $13 \%$ follow ring-deformation mechanisms (ring-opening and ring- 
puckering). $7 \%$ do not deactivate within the 200 fs of the dynamics simulation. Because of the uncertainties associated to the dynamics simulations and to the relatively small number of trajectories, these fractions should be taken as qualitative trends of occurrence of each mechanism, rather than a quantitative assessment of them. If trajectories starting in the $\pi \pi^{*}$ and in the $\pi \sigma_{\mathrm{NH}} *$ states are independently analyzed, these fractions remain essentially the same, implying that the population of each mechanism depends on the excitation energy, but not on the nature of the state. The fact that the fast $\mathrm{H}$ atom is formed along the $\mathrm{NH}$ stretching pathway either excited in the $\pi \pi^{*}$ or $\pi \sigma^{*}$ states has also been observed in the photofragment translational spectroscopy studies by Cronin et al. [26].

Experimental pump of pyrrole with $250 \mathrm{~nm}(4.96 \mathrm{eV})$ laser pulse followed by ionization probe with $241 \mathrm{~nm}(5.15 \mathrm{eV})$ pulse reveals two time constants, $\tau_{\mathrm{f}}=110 \pm 80 \mathrm{fs}$ and $\tau_{\mathrm{s}}=1100 \pm 500 \mathrm{fs}$ [19]. These time constants correspond to the time for formation of fast and slow $\mathrm{H}$ atoms, respectively. Since most of trajectories in our simulations finished in the ground state of the dissociated pyrrolyl $+\mathrm{H}$ system, the deactivation time $\tau_{0}$ should also approximately give the time for the formation of the fast $\mathrm{H}$ atoms population. Indeed, the comparison of $\tau_{0}$ and $\tau_{\mathrm{f}}$ shows good agreement. Note, however, that the initial state in the experiments (low energy $\pi \sigma_{\mathrm{NH}^{*}}$ ) and in the simulations (high energy $\pi \pi^{*}$ and $\pi \sigma_{\mathrm{NH}}{ }^{*}$ ) are not the same. This is an indication that the fast $\mathrm{H}$ elimination occurs directly by the same process, as soon as there is enough energy to overcome the $\pi 3 \mathrm{~s} / \pi \sigma_{\mathrm{NH}} *$ barrier in the $\mathrm{S}_{1}$ state.

Fig. 4 (around here)

Fig. 5 (around here)

The analysis of $\mathrm{NH}$ and $\mathrm{CN}$ bond distances was conducted for all trajectories and the results are presented in Fig. 5. The top panel of this figure shows that in some cases the CN distance is elongating during the dynamics. This behavior can be ascribed to the ring-opening 
and ring-puckering deactivation mechanisms. Since the main deactivation channel is the NHstretching, the majority of trajectories do not exhibit elongation of this specific bond. In the bottom panel of Fig. 5 the NH distance is monitored. In this figure three kinds of trajectories can be distinguished. For part of the trajectories the NH distance remain constant at about 1 $\AA$. They correspond to the trajectories following ring-distortion mechanisms. A minor fraction of trajectories (3) has the $\mathrm{NH}$ distance oscillating at a medium distance of about 2 or $3 \AA$. These are cases where the NH-stretching mechanism is activated, but instead finishing in dissociation, the hot ground state of pyrrole is formed. In most of the trajectories the $\mathrm{NH}$ distance is steadily increasing. In these cases, the $\mathrm{NH}$-stretching mechanism is activated and the $\mathrm{H}$ atom elimination is taking place. It should be mentioned that a cut-off value of $10 \AA$ for NH distance was used in Fig. 5 in order to simplify the data analysis. In some of the trajectories, however, the NH distance was longer, up to $40 \AA$.

Fig. 6 (around here)

Fig. 6a shows that the hydrogen dissociation starts on average 54 fs after the photoexcitation. The kinetic energy of the dissociated hydrogen atom has a broad distribution around the average value of $1.2 \mathrm{eV}$ (Fig. 6b). This value is in very good agreement with the experimental results, $\sim 1 \mathrm{eV}[11,26]$, for the center of the fast $\mathrm{H}$-elimination peak in the kinetic energy release spectra. Note that, as expected, there is no formation of a slow $\mathrm{H}$ elimination peak, which should take place in the picosecond timescale [19], much longer than the maximum simulation time (200 fs). The NH distance at the $\mathrm{S}_{1} \rightarrow \mathrm{S}_{0}$ hopping time is shown in Fig. 6c for all trajectories that have returned to the ground state. The histogram shows two distinct peaks. The first peak with average at $1.0 \AA$ will be discussed below. The second peak starts at $1.5 \AA$ and presents a long tail for large distances up to $4 \AA$. This peak corresponds to the trajectories deactivated by means of the NH-stretching mechanism. Its average value at 
$2.1 \AA$ is $0.2 \AA$ larger than the NH distance for the crossing between the lowest $\pi \sigma^{*}$ state and the ground state shown in Fig. 2 (left panel).

Fig. 7 (around here)

Twelve out of ninety trajectories did not follow the NH-stretching mechanism. They appear in the short-distance peak in Fig. 6c. In order to understand which kind of mechanism they followed, it is useful to project them on the Cremer-Pople (CP) space Q- $\phi$. This is shown in Fig. 7 for all structures for which the $S_{1}-S_{0}$ energy gap is smaller than $0.5 \mathrm{eV}$ (open dots) and for structures at the hopping time (full dots). The ring-opened MXS is at $\mathrm{Q}=0 \AA$ and the ring-puckered MXS is shown by a cross ( $E_{1}$ conformation). Since the ring-opened and the ring-puckered conical intersections correspond to distinct types of structures on the crossing seam with different electronic configurations, it could be expected that the structures at the hopping time would cluster in two disjoint regions around these MXSs. This, however, is not the case. Fig. 7 shows that the non-adiabatic events occur in a large continuous portion of the $\mathrm{CP}$ space, indicating that the crossing seam spans this entire region. The degree of puckering varies from almost planar $(Q=0.15 \AA)$ to the strongly puckered structures $(Q=0.75 \AA)$. Most of hopping events occur at $\mathrm{E}_{1},{ }^{2} \mathrm{~T}_{1}$ and ${ }^{2} \mathrm{E}$ conformations, indicating that not only the $\mathrm{E}_{1}$ conformation of the ring-puckered MXS, but also other kinds of puckering can give rise to conical intersections in pyrrole.

If we take $\mathrm{Q}=0.3 \AA$ as an arbitrary threshold to distinguish between the ring-opening and ring-puckering mechanisms, nine trajectories deactivated at ring-puckered conformations and three trajectories deactivated at ring-opened conformations, thus corresponding to $10 \%$ and $3 \%$ of the total number of trajectories, respectively (see Fig. 4). 


\section{Conclusions}

The photochemical processes in pyrrole were investigated using a high-level multireference configuration interaction method (MRCI) giving a balanced description of the four studied excited states, two of Rydberg character and two valence states. Cuts along the potential energy surfaces connecting the Franck-Condon region and three different minima on the crossing seam (MXS) (NH dissociation, ring puckering, and a planar ring-opened MXS) describe possible deactivation pathways. One of these intersection points, the ring-opened MXS, was characterized for the first time. Although it is the conical intersection of the lowest energy identified in pyrrole so far, its efficiency for the internal conversion process seems to be reduced by the required strong geometric deformations and by the diabatic change of the initially excited $\pi \pi^{*}$ state into the $\pi \sigma_{N C} *$ state, which in turn crosses the ground state.

Non-adiabatic surface-hopping dynamics simulations of pyrrole were performed for 200 fs starting in the $S_{4}$ state and using a high-level MR-CI approach for the electronic structure calculations. The dynamics simulations show that in fact all three types of conical intersections were accessed. The transfer of population from the initially excited $\mathrm{S}_{4}$ state to the ground state takes place in about 140 fs. This process occurs basically in two steps, with the $S_{1}$ state being populated in about $44 \mathrm{fs}$ and then being depleted in about $80 \mathrm{fs}$. Most of trajectories $(80 \%)$ dissociated rapidly along the repulsive $\pi \sigma_{\mathrm{NH}^{*}}$ state giving rise to a population of fast $\mathrm{H}$ atoms. The computed deactivation time of 140 fs agrees very well with the experimentally measured time constant of $110 \mathrm{fs}$ for the formation of fast hydrogen atoms. The computed average kinetic energy agrees very well with the experimentally observed average kinetic energy of the fast hydrogen atoms. A fraction of $13 \%$ of trajectories follows ring-deformation channels involving either ring puckering (10\%) or planar ring opening (3\%). These fractions did not depend on whether the initial state had $\pi \pi^{*}$ or $\pi \sigma_{\mathrm{NH}} *$ character. 
Our calculations provide a detailed picture of the photodeactivation processes in pyrrole. Although the main objective of this work - the observation of the occurrence of the different deactivation mechanisms - has been accomplished, it should be noted that the participation of $\pi \sigma_{\mathrm{NH}} *$ states in the initial conditions was much higher than what would be expected from the oscillator strengths of these two transitions. This bias occurred because of the relatively high vertical excitation energy of the ${ }^{1} \mathrm{~B}_{1}$ Rydberg state, which caused frequent exchange of position with the $\pi \pi^{*}$ in the Wigner sample. Interestingly, it turned out that the observed percentages of the different mechanisms was insensitive to the initial character of $\mathrm{S}_{4}$, consequently implying that that this bias is not so critical for the general interpretations. Nevertheless, more investigations are needed to analyze the influence of excitation energies on the product yields in order to explain the experimentally observed strong energy dependence of the branching ratios for fast and slow hydrogen atoms.

\section{Acknowledgments}

This work was supported by the Austrian Science Fund within the framework of the Special Research Program F16 (Advanced Light Sources) and Project P18411-N19. The calculations were partially performed at the Linux PC cluster Schrödinger III of the computer center of the University of Vienna. The work in Zagreb (M.E.M and M.V.) was supported by the Ministry of Science, Education and Sport through the project 098-0982933-2920 and the COST D37 action.

\section{References}

[1] L. Serrano-Andrés, M. Merchán, I. Nebotgil, B. O. Roos, and M. Fulscher, J. Am. Chem. Soc. 115, 6184 (1993).

[2] M. H. Palmer, I. C. Walker, and M. F. Guest, Chem. Phys. 238, 179 (1998). 
[3] O. Christiansen, J. Gauss, J. F. Stanton, and P. Jorgensen, J. Chem. Phys. 111, 525 (1999).

[4] D. J. Tozer, R. D. Amos, N. C. Handy, B. O. Roos, and L. Serrano-Andres, Mol. Phys. 97, 859 (1999).

[5] J. Wan, J. Meller, M. Hada, M. Ehara, and H. Nakatsuji, J. Chem. Phys. 113, 7853 (2000).

[6] B. O. Roos, P. A. Malmqvist, V. Molina, L. Serrano-Andres, and M. Merchan, J. Chem. Phys. 116, 7526 (2002).

[7] C. G. Zhan, and D. A. Dixon, J. Mol. Spectrosc. 216, 81 (2002).

[8] P. Celani, and H. J. Werner, J. Chem. Phys. 119, 5044 (2003).

[9] M. H. Palmer, and P. J. Wilson, Mol. Phys. 101, 2391 (2003).

[10] M. Pastore, C. Angeli, and R. Cimiraglia, Chem. Phys. Lett. 422, 522 (2006).

[11] D. A. Blank, S. W. North, and Y. T. Lee, Chem. Phys. 187, 35 (1994).

[12] A. B. Trofimov, H. Köppel, and J. Schirmer, J. Chem. Phys. 109, 1025 (1998).

[13] A. L. Sobolewski, W. Domcke, C. Dedonder-Lardeux, and C. Jouvet, PCCP 4, 1093 (2002).

[14] J. Wei, A. Kuczmann, J. Riedel, F. Renth, and F. Temps, PCCP 5, 315 (2003).

[15] V. Vallet, Z. G. Lan, S. Mahapatra, A. L. Sobolewski, and W. Domcke, Faraday Discuss. 127, 283 (2004).

[16] V. Vallet, Z. G. Lan, S. Mahapatra, A. L. Sobolewski, and W. Domcke, J. Chem. Phys. 123 (2005).

[17] J. Wei, J. Riedel, A. Kuczmann, F. Renth, and F. Temps, Faraday Discuss. 127, 267 (2004).

[18] H. Köppel, E. V. Gromov, and A. B. Trofimov, Chem. Phys. 304, 35 (2004).

[19] H. Lippert, H. H. Ritze, I. V. Hertel, and W. Radloff, Chemphyschem 5, 1423 (2004).

[20] A. J. van den Brom, M. Kapelios, T. N. Kitsopoulos, N. H. Nahler, B. Cronin, and M. N. R. Ashfold, PCCP 7, 892 (2005).

[21] I. Frank, and K. Damianos, Journal of Chemical Physics 126 (2007). 
[22] Z. Lan, A. Dupays, V. Vallet, S. Mahapatra, and W. Domcke, Journal of Photochemistry and Photobiology a-Chemistry 190, 177 (2007).

[23] Z. Lan, and W. Domcke, Chem. Phys. 350, 125 (2008).

[24] A. Kumar, M. Kolaski, and K. S. Kim, J. Chem. Phys. 128 (2008).

[25] E. J. Shin, Bull. Korean Chem. Soc. 25, 907 (2004).

[26] B. Cronin, M. G. D. Nix, R. H. Qadiri, and M. N. R. Ashfold, PCCP 6, 5031 (2004).

[27] M. Barbatti, M. Vazdar, A. J. A. Aquino, M. Eckert-Maksic, and H. Lischka, J. Chem. Phys. 125, 164323 (2006).

[28] V. Poterya, V. Profant, M. Farnik, P. Slavicek, and U. Buck, J. Chem. Phys. 127, 064307 (2007).

[29] M. Barbatti, B. Sellner, A. J. A. Aquino, and H. Lischka, in Radiation Induced Molecular Phenomena in Nucleic Acid, edited by M. K. Shukla, and J. Leszczynski (Springer, Netherlands, 2008), pp. 209.

[30] S. Salzmann, M. Kleinschmidt, J. Tatchen, R. Weinkauf, and C. M. Marian, PCCP 10, 380 (2008).

[31] N. Gavrilov, S. Salzmann, and C. M. Marian, Chem. Phys. 349, 269 (2008).

[32] M. Barbatti, H. Lischka, S. Salzmann, and C. M. Marian, J. Chem. Phys., submitted (2008).

[33] S. Perun, A. L. Sobolewski, and W. Domcke, Chem. Phys. 313, 107 (2005).

[34] M. Barbatti, and H. Lischka, J. Am. Chem. Soc. 130, 6831 (2008).

[35] V. Profant, V. Poterya, M. Farnik, P. Slavicek, and U. Buck, J. Phys. Chem. A 111, 12477 (2007).

[36] A. L. Sobolewski, and W. Domcke, Chem. Phys. 259, 181 (2000).

[37] I. Antol, M. Vazdar, M. Barbatti, and M. Eckert-Maksic, Chem. Phys. 349, 308 (2008).

[38] M. Barbatti, M. Ruckenbauer, J. J. Szymczak, A. J. A. Aquino, and H. Lischka, PCCP 10, 482 (2008). 
[39] J. C. Tully, Faraday Discuss. 110, 407 (1998).

[40] A. Ferretti, G. Granucci, A. Lami, M. Persico, and G. Villani, J. Chem. Phys. 104, 5517 (1996).

[41] N. L. Doltsinis, and D. Marx, Phys. Rev. Lett. 88, 166402 (2002).

[42] M. Barbatti, G. Granucci, M. Persico, M. Ruckenbauer, M. Vazdar, M. Eckert-Maksic, and H. Lischka, J. Photochem. Photobiol., A 190, 228 (2007).

[43] E. Fabiano, T. W. Keal, and W. Thiel, Chem. Phys. 349, 334 (2008).

[44] J. Pittner, H. Lischka, and M. Barbatti, Chem. Phys., doi:10.1016/j.chemphys.2008.10.013 (2008).

[45] J. C. Tully, J. Chem. Phys. 93, 1061 (1990).

[46] L. Sun, and W. L. Hase, in Reviews in Computational Chemistry, edited by K. B. Lipkowitz et al. (Wiley-VCH, New York, 2003), pp. 79.

[47] H. Lischka, R. Shepard, F. B. Brown, and I. Shavitt, Int. J. Quantum Chem. S.15, 91 (1981).

[48] H. Lischka, R. Shepard, R. M. Pitzer, I. Shavitt, M. Dallos, T. Müller, P. G. Szalay, M. Seth, G. S. Kedziora, S. Yabushita, and Z. Y. Zhang, PCCP 3, 664 (2001).

[49] H. Lischka, R. Shepard, I. Shavitt, R. M. Pitzer, M. Dallos, T. Mueller, P. G. Szalay, F. B. Brown, R. Ahlrichs, H. J. Boehm, A. Chang, D. C. Comeau, R. Gdanitz, H. Dachsel, C. Ehrhardt, M. Ernzerhof, P. Hoechtl, S. Irle, G. Kedziora, T. Kovar, V. Parasuk, M. J. M. Pepper, P. Scharf, H. Schiffer, M. Schindler, M. Schueler, M. Seth, E. A. Stahlberg, J.-G. Zhao, S. Yabushita, Z. Zhang, M. Barbatti, S. Matsika, M. Schuurmann, D. R. Yarkony, S. R. Brozell, E. V. Beck, and J.P. Blaudeau, COLUMBUS, an ab initio electronic structure program, release 5.9.1, www.univie.ac.at/columbus (2006).

[50] A. Bunge, J. Chem. Phys. 53, 20 (1970).

[51] T. H. Dunning, J. Chem. Phys. 90, 1007 (1989). 
[52] R. Shepard, H. Lischka, P. G. Szalay, T. Kovar, and M. Ernzerhof, J. Chem. Phys. 96, 2085 (1992).

[53] R. Shepard, in Modern Electronic Structure Theory, edited by D. R. Yarkony (World Scientific, Singapore, 1995), p. 345.

[54] H. Lischka, M. Dallos, and R. Shepard, Mol. Phys. 100, 1647 (2002).

[55] M. Dallos, H. Lischka, R. Shepard, D. R. Yarkony, and P. G. Szalay, Journal of Chemical Physics 120, 7330 (2004).

[56] H. Lischka, M. Dallos, P. G. Szalay, D. R. Yarkony, and R. Shepard, Journal of Chemical Physics 120, 7322 (2004).

[57] S. R. Langhoff, and E. R. Davidson, Int. J. Quantum Chem. 8, 61 (1974).

[58] P. J. Bruna, S. D. Peyerimhoff, and R. J. Buenker, Chem. Phys. Lett. 72, 278 (1980).

[59] V. Bonacic-Koutecky, and R. Mitric, Chem. Rev. 105, 11 (2005).

[60] R. Mitric, V. Bonacic-Koutecky, J. Pittner, and H. Lischka, J. Chem. Phys. 125 (2006).

[61] W. C. Swope, H. C. Andersen, P. H. Berens, and K. R. Wilson, J. Chem. Phys. 76, 637 (1982).

[62] G. Granucci, and M. Persico, J. Chem. Phys. 126, 134114 (2007).

[63] D. Cremer, and J. A. Pople, J. Am. Chem. Soc. 97, 1354 (1975).

[64] T. Helgaker, H. J. A. Jensen, P. Jørgensen, J. Olsen, K. Ruud, H. Ågren, T. Andersen, K. L. Bak, V. Bakken, O. Christiansen, P. Dahle, E. K. Dalskov, T. Enevoldsen, H. Heiberg, H. Hettema, D. Jonsson, S. Kirpekar, R. Kobayashi, H. Koch, K. V. Mikkelsen, P. Norman, M. J. Packer, T. Saue, P. R. Taylor, and O. Vahtras, DALTON, an ab initio electronic structure program, Release 1.0 (1997).

[65] M. Barbatti, G. Granucci, M. Ruckenbauer, M. Persico, and H. Lischka, NEWTON-X: a package for Newtonian dynamics close to the crossing seam, www.univie.ac.at/newtonx (2007). 


\section{Tables}

Table 1 - Vertical excitation energies of selected singlet states of pyrrole.

\begin{tabular}{llcccccc}
\hline \multicolumn{2}{l}{ State } & MRCI $^{\mathrm{a}}$ & MRCI+Q $^{\mathrm{b}}$ & TDDFT $^{\mathrm{c}}$ & $\mathrm{CASPT}^{2}$ & $\mathrm{CC}^{\mathrm{g}}$ & Exp \\
\hline $\mathrm{S}_{0}$ & ${ }^{\mathrm{l}} \mathrm{A}_{1}$ & 0.00 & 0.00 & 0.00 & 0.00 & 0.00 & \\
$\pi 3 \mathrm{~s}$ & ${ }^{1} \mathrm{~A}_{2}$ & 5.22 & 5.09 & 5.05 & $5.22^{\mathrm{d}} / 5.22^{\mathrm{e}}$ & 5.10 & $5.08^{\mathrm{h}} / 5.22^{\mathrm{i}}$ \\
$\pi 3 \mathrm{~s}$ & ${ }^{1} \mathrm{~B}_{1}$ & 6.21 & 5.86 & 5.88 & $5.85^{\mathrm{f}} / 5.92^{\mathrm{e}}$ & 5.99 & $6.22^{\mathrm{h}}$ \\
$\pi \pi^{*}$ & ${ }^{1} \mathrm{~A}_{1}$ & 6.55 & 6.39 & 6.29 & $5.82^{\mathrm{d}} / 5.98^{\mathrm{e}}$ & 6.37 & \\
$\pi \pi^{*}$ & ${ }^{1} \mathrm{~B}_{2}$ & 6.65 & 6.78 & 6.45 & $5.87^{\mathrm{d}} / 5.95^{\mathrm{e}}$ & 6.63 & $5.92^{\mathrm{h}} / 6.2-6.4^{\mathrm{i}}$ \\
\hline
\end{tabular}

${ }^{a}$ Present results, MR-CISD/SA-5-CASSCF(4,5)/BS

${ }^{\mathrm{b}}$ MR-CISD+Q/SA-5-CAS(6,5)+AUX(1)/d-aug-cc-pVDZ, Reference [27].

${ }^{\mathrm{c}}$ Reference [4].

${ }^{\mathrm{d}}$ Reference [6].

${ }^{\mathrm{e}}$ Reference [8].

${ }^{\mathrm{f}}$ Reference [1].

${ }^{\mathrm{g}}$ Reference [3].

${ }^{\mathrm{h}}$ Assigments given in Reference [9].

${ }^{\mathrm{i}}$ Assigments given in Reference [3]. 
Table 2- Energy of pyrrole MXSs (in eV) relative to the minimum in the ground state.

\begin{tabular}{llllll}
\hline MXS & MRCI $^{\mathrm{a}}$ & MRCI+Q $^{\mathrm{a}}$ & MRCI & MRCI+Q & MXS features \\
\hline$\pi \pi * / \mathrm{S}_{0}\left(\mathrm{E}_{1}\right)$ & 4.95 & 4.86 & $4.89^{\mathrm{b}}$ & $4.93^{\mathrm{b}}$ & ring puckering, Fig. 1a \\
$\pi \sigma_{\mathrm{NH}} * / \mathrm{S}_{0}$ & 4.45 & 4.26 & $4.41^{\mathrm{c}}$ & $4.44^{\mathrm{c}}$ & NH stretching, Fig. 1b \\
$\pi \sigma_{\mathrm{NC}} / \mathrm{S}_{0}$ & 4.11 & 3.86 & - & - & ring opening, Fig. 1c \\
\hline
\end{tabular}

${ }^{a}$ Present results, MR-CISD/SA-5-CASSCF(4,5)/BS

${ }^{b}$ MR-CISD(Q)/SA-3-CAS(6,5)/6-31G(d), Reference [27].

${ }^{c}$ MR-CISD(Q)/SA-3-CAS(6,6)/6-31G(d), Reference [27]. 


\section{Figure Captions}

Fig. 1. Structures and selected geometric parameters for pyrrole MXSs obtained at the MRCI level. Distances are given in $\AA$ and dihedral angles in degrees. The number in brackets correspond to the benchmark MRCI value from Ref. [27].

Fig. 2. Potential energy curves calculated at the MRCI level along a) the rigid NH-stretching coordinate and along the LIIC path from the ground state minimum to b) the ring-puckered MXS and to c) the ring-opened MXS.

Fig. 3. Average adiabatic populations of trajectories for each state as a function of time after initial photoexcitation of pyrrole into the $\mathrm{S}_{4}$ state.

Fig. 4. Description and statistics of trajectory deactivation mechanisms.

Fig. 5. CN (top) and $\mathrm{NH}$ (bottom) distance variations as a function of time for all trajectories. The NH distance of $10 \AA$ was used as a cut-off value (see text).

Fig. 6. Analysis of the trajectories showing NH dissociation. (a) Initial time of the dissociation, taking $2 \AA$ for the $\mathrm{NH}$ bond as reference value. (b) Hydrogen kinetic energy. (c) NH distance for all trajectories at the time of the $\mathrm{S}_{1} \rightarrow \mathrm{S}_{0}$ hopping.

Fig. 7. Distribution of conformations in the Cremer-Pople Q- $\phi$ space for trajectories following ringdeformation mechanisms. Full dots: conformations at the hopping time. Open dots: conformations with $\mathrm{S}_{1}-\mathrm{S}_{0}$ energy gaps smaller than $0.5 \mathrm{eV}$. Cross: ring puckered MXS. 